\title{
Density dependent environments can select for extremes of body size
}

\author{
T. Coulson ${ }^{1, *}$ \\ A. Felmy ${ }^{2}$ \\ T. Potter ${ }^{3}$ \\ G. Passoni ${ }^{1}$ \\ R.A. Mongomery ${ }^{1}$ \\ J.M. Gaillard ${ }^{5}$ \\ P.J. Hudson ${ }^{4}$ \\ J. Travis ${ }^{3}$ \\ R.D. Bassar ${ }^{6}$ \\ S. Tuljapurkar ${ }^{7}$ \\ D.J. Marshall ${ }^{8}$ \\ S.M. Clegg ${ }^{1}$
}

18 February 2022

\begin{abstract}
Body size variation is an enigma. We do not understand why species achieve the sizes they do, and this means we also do not understand the circumstances under which gigantism or dwarfism is selected. We develop size-structured integral projection models to explore evolution of body size and life history speed. We make few assumptions and keep models simple: all functions remain constant across models except for the one that describes development of body size with age; size at sexual maturity is constant at $80 \%$ of asymptotic size across life histories; and density-dependence impacts only reproduction or juvenile survival. Carrying capacity is fitness in the models we develop and we are consequently interested in how it changes with size at sexual maturity, and how this association varies with development rate. The simple models generate complex dynamics while providing insight into the circumstances when extremes of body size evolve. We identify different areas of parameter space where gigantism and dwarfism evolve. The direction of selection depends upon emergent trade-offs among the proportion of each cohort that survives to sexual maturity, life expectancy at sexual maturity, and the per-capita reproductive rate. The specific trade-offs that emerge depend upon where density-dependence operates in the life history. Empirical application of the approach we develop has potential to help explain the enigma of body size
\end{abstract}



variation across the tree of life.

${ }^{1}$ Department of Biology, University of Oxford, Oxford, OX1 3SZ, UK

${ }_{20} \quad{ }^{2}$ Department of Evolutionary Biology and Environmental Studies, University of Zurich, Switzerland

${ }_{21} \quad{ }^{3}$ Department of Biological Science, Florida State University, Tallahassee FL 32306, USA

${ }_{22} 4$ The Huck Institutes, Penn State University, State College, PA 16802, USA

${ }_{23} \quad{ }^{5}$ Laboratoire de Biometrie et Biology Evolutive, University of Lyon 1, Lyon, France

${ }_{24}{ }^{6}$ Department of Biology, Williams College, Williamstown, MA 01267, USA

${ }_{25}{ }^{7}$ Department of Biology, Stanford University, Palo Alto, CA 94305, USA

${ }_{26}{ }^{8}$ School of Biological Sciences, Monash University, Melbourne, Victoria, Australia 3800

${ }_{27}^{*}$ Correspondence: T. Coulson $<$ tim.coulson@zoo.ox.ac.uk> 


\section{Introduction}

Stout infantfish (Schindleria brevipinguis) achieve sexual maturity at less than 0.1g (Watson \& Walker 2004) while blue whales (Balaenoptera musculus) can grow to weigh 150 tonnes representing a span in adult weights of over nine orders of magnitude. Lifespan in vertebrates is not quite so variable, but the range of three orders of magnitude is still impressive: Greenland sharks (Somniosus microcephalus) can live up to half a millennium (Nielsen et al. 2016), while the coral reef fish, the seven-figure pygmy goby (Eviota sigillata), is elderly if it survives for two months (Depczynski \& Bellwood 2005). There are clearly physiological limits that define the extremes of body size and longevity in vertebrates, but the factors that may push organisms towards these extremes are presently unclear.

Body size evolution, particularly when resulting in either gigantism or dwarfism, has long fascinated biologists. Body size variation across species is statistically associated with life history variation in an allometric manner (West et al. 1997; Savage et al. 2004). As size increases, there is also an increase in the value of traits measured in units of mass (e.g. neonatal mass), time (e.g. life expectancy), and length (e.g. body length). In contrast, as size increases, the values of traits describing the frequency of events, such as reproductive rates, decrease.

Within species, patterns of size variation are less clear. Body size is regularly measured within populations, is often under directional selection, but rarely evolves in line with predictions (Kingsolver et al. 2001; Merilä et al. 2001). Body size evolution remains challenging to predict because identical processes can result in increases in body size and a slowing of the life history in some species, yet the exact opposite in others: food limitation selects for an increase in body size at sexual maturity in some species of fish (Travis et al. 2014), but a decrease in ungulates (Raia \& Meiri 2006). Body size variation is an enigma, and we do not have a good understanding of why species are the size they are (Audzijonyte et al. 2020).

Darwinian demons are hypothetical creatures capable of simultaneously maximizing all components of fitness (Law 1979). In doing so, they achieve sexual maturity immediately after birth, continuously produce litter sizes of an infinite number of viable young, and are immortal. They would presumably be tiny, perhaps infinitesimally so, given development takes time. Regardless of their size, we would instantaneously be neck-deep in such pests. Fortunately, Darwinian demons do not exist because all individuals face trade-offs.

In the absence of trade-offs, populations are expected to grow exponentially and organisms are expected to evolve towards a Darwinian demon life history as allocation of resources to early reproduction is always favored under such circumstances (McGraw \& Caswell 1996; Coulson et al. 2006). Trade-offs occur when something prevents all components of fitness being maximized simultaneously (Stearns 1977; Stearns 1992; Kozłowski et al. 2020). Energy availability is frequently assumed to be the constraint that generates trade-offs (Kooijman \& Kooijman 2010), but the availability of enemy-free space, breeding sites, water, or other molecules essential for life, can also generate them. Trade-offs consequently arise when something limits population growth. The question we are interested in 
is how trade-offs can select for long developmental periods, large body size, and slow life histories, the apparent antithesis of Darwinian demons?

Approaches to understanding both life history and body size evolution often involve specifying a limiting factor and a life history trade-off, before identifying the fittest strategy. For example, in bioenergetic and dynamic energy budget models, energy is assumed to be limiting, the trade-off is specified via rules determining the allocation of energy to maintenance, development, and reproduction (Kooijman \& Kooijman 2010), and the fittest strategy is identified, usually via an evolutionary game (Kozłowski 1992; Day \& Taylor 1997; Koziowski \& Weiner 1997). A related approach involves agnosticism as to the limiting factor, a priori specification of a trade-off between two components of the life history such as offspring number and offspring size (Smith \& Fretwell 1974), and use of an evolutionary game to identify the fittest strategy (Roff 1993; Grant 1997; Meszena et al. 2002; Childs et al. 2004; Metcalf et al. 2008). A third alternative is to identify the quantity that evolution maximizes (e.g., fitness), and to examine how independently altering each part of the life history impacts fitness. Selection is then assumed to predominantly operate on the components with the largest sensitivities of fitness (Caswell 2001; Tuljapurkar et al. 2009; Jones \& Tuljapurkar 2015). This approach has been used for deterministic, density-independent environments where fitness is the population growth rate $r$, and stochastic, density-independent environments where fitness is the long-run stochastic growth rate $a$ (Tuljapurkar et al. 2009).

The third approach has the virtue of making few assumptions as it does not require specification of a trade-off, but it does suffer from a shortcoming in that continuous population growth occurs in the absence of population limitation, and therefore environmentally-determined trade-offs shaping evolutionary trajectories may not exist. We address this limitation by using the approach in density-dependent environments where population limitation, by definition, exists (Turchin 1999). This imposes a constraint on population growth and mean lifetime reproductive success $R 0$, but does not require us to make any assumptions about the cause of the limiting factor (e.g. energy or enemy-free space), and we do not need to specify a trade-off a priori. Instead, the trade-offs emerge as a function of where in the life cycle limiting processes operate most strongly, and where they are absent (see also Charlesworth 1994).

When trade-offs reveal themselves via the imposition of population limitation, population size will achieve an equilibrium referred to as carrying capacity $K$, density-dependence will be observed, and the population growth rate will equal zero. It is tempting to equate density-dependence with food limitation (White 2008), but that is too narrow a definition. Density dependence is simply a statistical pattern where no long-term temporal trend in population numbers is observed. Any limiting resource can generate density-dependent dynamics (Turchin 1999). In deterministic, density-dependent environments, regardless of the limiting process, fitness is carrying capacity (Lande et al. 2009; Sæther \& Engen 2015; Kentie et al. 2020). It is helpful to briefly explain how to interpret this statement.

Fitness is genetic representation of a heritable entity (be it an allele, genotype, phenotype, or strategy), either expected (Charlesworth 1994) or realized (Coulson et al. 2006), in a population at some point in the future. 
Given future genetic representation will depend upon how quickly the heritable entity replicates and the degree of heritability (Fisher 1930), fitness can also be thought of as a growth rate, such as reproductive value (Grafen 1999), or how quickly the entity can invade a population (Dieckmann et al. 2006). When carrying capacity is said to be fitness, it is shorthand for carrying capacity being able to predict future asymptotic representation within a population, and whether one heritable entity would replace another in an evolutionary game (Kentie et al. 2020). For example, consider competing strategies and assume that strategy $A$ has a carrying capacity of $X$ and strategy $B$ of $X-q$. If one individual of strategy $B$ were introduced into a population of strategy $A$ at its carrying capacity $X$, it could not establish, because it would experience a population density that is greater than its carrying capacity and its replacement rate $\lambda_{B}$, and its mean lifetime reproductive success $R 0_{B}$ would both be less than unity. In contrast, if the experiment were repeated the other way around, strategy $A$ would have a growth rate $\lambda_{A}>1$ and $R 0_{A}>1$ because it would be introduced into a population below its own carrying capacity (Meszena et al. 2002; Childs et al. 2004; Dieckmann et al. 2006). If we know the carrying capacities of strategies $A$ and $B$, we do not need to run an evolutionary game to identify the evolutionarily stable strategy (Kentie et al. 2020).

Since we know what fitness is in a density-dependent environment, we can use an approach related to those used for density-independent environments where we know what quantity evolution maximizes (Caswell 2001; Tuljapurkar et al. 2009; Jones \& Tuljapurkar 2015). Because we are interested in understanding the evolution of extremes of body size, we develop size-structured models (that are density-dependent), and we examine how altering growth trajectories impacts body size and life history evolution while imposing a constraint that sexual maturity occurs at a fixed proportion of asymptotic size. We discover that:

1. Delaying sexual maturity can generate a demographic cost to juveniles, such that a smaller proportion of each cohort survives to maturity. If this cost is more than offset by a demographic benefit to adults, via either an increase in life expectancy or increased reproduction, then larger body sizes and slower life history strategies will be selected. If the juvenile fitness cost is not offset by the adult fitness benefit, then small body sizes and faster life histories will evolve.

2. Because carrying capacity is fitness in the models we develop, population growth and mean lifetime reproductive success both equal unity at equilibrium. Evolution acts to maximize population size, resulting in an inevitable reduction in the value of demographic rates influenced negatively by density-dependence for individuals of a given body size. As density-dependent demographic rates are minimized, those that are not density-dependent are maximized.

3. The simultaneous minimization of density-dependent rates and maximization of density-independent rates generates life history trade-offs. Where in the life history these trade-offs occur depends upon which demographic rates are influenced by negative feedbacks, and which are not. 


\section{${ }_{125}$ Methods}

We deliberately make few assumptions, and strive to keep models simple, while choosing forms of demographic functions that are typical of those observed in nature such as an increase in survival rate with body size, and a juvenile and adult stage either side of sexual maturity. Terms used in the text are defined in Table 1.

\section{The model}

We develop a size-structured integral projection model (IPM) (Coulson 2012; Ellner et al. 2016) that consists of four equations describing the association between body size $z$ at time $t$ and i) survival to time $t+1, S(z, N, t)=$ $\frac{1}{1+e^{\left.-\left(\beta_{0}+\beta_{z} z+\beta_{N} N(t)\right)\right)}}$, ii) development of surviving individuals from $t$ to $t+1, G\left(z^{\prime} \mid z, N, t\right)=\theta\left(\mu=\alpha_{0}+\alpha_{z} z+\right.$ $\left.\alpha_{N} N(t), \sigma^{2}=\alpha_{v}\right)$, iii) the per-capita reproductive rate between $t$ and $t+1$,

$$
R(z, N, t)= \begin{cases}0, & \text { if } z<z_{a} \\ e^{\rho_{0}+\rho_{z} z+\rho_{N} N(t)}, & \text { otherwise }\end{cases}
$$

and iv) the body size of these offspring at recruitment to the population at $t+1, D\left(z^{\prime} \mid z, N, t\right)=\theta\left(\mu=\gamma_{0}, \sigma^{2}=\gamma_{v}\right)$ where the $\theta \mathrm{s}$ are normal distributions with means of $\mu$ and variances $\sigma^{2}$, and the $\alpha \mathrm{s}, \beta \mathrm{s}, \gamma \mathrm{s}$ and $\rho \mathrm{s}$ are parameters, and $z_{a}$ is size at sexual maturity. These four functions combine to iterate forward the distribution of body size $N(z, t)$ within the population at time $t$ to the distribution of body size $N\left(z^{\prime}, t+1\right)$ at time $t+1$ :

$$
N\left(z^{\prime}, t+1\right)=\int\left(D\left(z^{\prime} \mid z, N, t\right) R(z, N, t)+G\left(z^{\prime} \mid z, N, t\right) S(z, N, t)\right) N(z, t) d z .
$$

The function $G\left(z^{\prime} \mid z, N, t\right)$ is called the development function as is standard nomenclature in IPM notation (Coulson et al. 2017), and, in the models we develop, describes growth from one age to the next. IPMs can be constructed for any continuous phenotypic trait - not just body size - and the function can be mechanistic, capturing detailed developmental pathways, or phenomenological based on repeated phenotypic measurements taken on the same individuals over time (Ellner et al. 2016; Smallegange et al. 2017; Lachish et al. 2020).

Because this is a density-dependent model, at equilibrium $N(z, t)=N\left(z^{\prime}, t+1\right)$. We discretise each of the functions to allow us to approximate the integral projection model in matrix form using standard approaches (Ellner et al. 2016). At equilibrium, the approximation is $\mathbf{K}=(\mathbf{D R}+\mathbf{G S}) \mathbf{K}$ where $\mathbf{K}$ is a vector describing the population structure at carrying capacity.

For simplicity, we only include density-dependence in one function at a time. In the first scenario, reproduction is density-dependent and $\rho_{N}<0$, while in the second, the density-dependent demographic rate is juvenile survival. In the second scenario, 


$$
S(z, N, t)= \begin{cases}\beta_{N}<0, & \text { if } z<z_{a} \\ \beta_{N}=0, & \text { otherwise }\end{cases}
$$

At equilibrium, when the population size of a life history is at carrying capacity, both the population growth rate $\lambda$ and mean lifetime reproductive $R 0$ are equal to unity - the dominant eigenvalue of the matrix approximation is 1 . Our analysis proceeds by iterating a population with a given life history strategy through time until it reaches $K$. We then report dynamics at $K$ for each life history. We compare across life histories to draw conclusions on the direction of evolution.

\section{Defining life history strategies}

Within each of the two scenarios, we construct 20 models, with each model representing a different life history strategy. Within a scenario, each of these 20 models has identical parameter values for each function, with the exception of the development function $G\left(z^{\prime} \mid z, N, t\right)$ and the size at sexual maturity $z_{a}$ which is always $80 \%$ of asymptotic size. Different parameterisations of the development function generate different stable size distributions (the dominant right eigenvector of the IPM evaluated at $K$ ) for each life history, and these differences generate variation in age-specific survival rates (see below). Demographic rates must combine to give $\lambda=R 0=1$ at equilibrium. Because survival rates vary across life history in both scenarios, the one degree of freedom available within the model to satisfy the condition $\lambda=R 0=1$ at equilibrium is the value of $K$ in the density-dependent function (reproduction in scenario 1, juvenile survival in scenario 2). For each model, we find the value of $K$ via simulation. The life history with the largest value of $K$ will be the fittest, and in an evolutionary game would grow to dominate the population.

We keep the models simple by assuming that each reproducing parent produces the same distribution of offspring body sizes regardless of their size or life history strategy (Figure 1(A)). We also assume that all offspring develop at the same pace over the first year of life regardless of life history strategy. After that age, the development functions diverge among the life histories (Figure 1(B)), such that those that will go on to achieve a larger size and greater age at sexual maturity continue to develop quickly, while those that will mature at a smaller size and lesser age slow their growth rates, reaching their asymptotic sizes at a younger age (Figure 1(C)). The growth models are monomolecular, such that growth rate slows with increasing size (Table 2, growth parameters). We choose this formulation because (i) monomolecular growth is a good descriptor of growth in many species, and (ii) it requires fewer parameters than non-linear growth forms (Gaillard et al. 1997; English et al. 2012). It is possible that a change in size at sexual maturity as life histories evolve will result in change in the form of the development function, for example, from monomolecular to logistic. Such a change might reflect, for example, individuals evolving to grow more slowly but for longer to achieve a larger size at sexual maturity as in island birds (Sandvig et al. 2019), but we 
do not consider functional forms of growth other than monomolecular here.

Survival rates increase with body size in all life histories in the same manner (Figure 1(D)), although when densitydependence operates on juvenile survival in the second scenario this function is depressed below $z<z_{a}$ for each life history (see below). Reproduction does not vary with size, i.e. $\rho_{z}=0$ in both scenarios, but the elevation of the function does vary with population density in the first scenario where reproduction is density dependent. Parameter values slightly differ for the two scenarios to enable us to more easily explain the observed dynamics, and are presented in Table 2.

\section{Exploring Model Dynamics}

We address two complementary questions: 1) How does maximizing $\mathrm{K}$ lead to the evolution of extreme body sizes and life histories? 2) How does the evolution of extreme body sizes and life histories translate into demographic patterns that maximize carrying capacity? To do this, we need to examine the population dynamics and life history the model describes. Because the model distinguishes juveniles $z<z_{a}$ and adults $z \geq z_{a}$, we find it useful to write the population dynamics and life history the models predict in terms of juvenile and adult rates. In this section, we describe some initial insights from the model structure, and explain how we extract the dynamics in terms of juvenile and adult rates.

One thing is immediately apparent from the model structure: as carrying capacity increases across life histories, the predicted value of the density-dependent rates will decrease all other things being equal. For example, in scenario 1 where reproduction is density-dependent, the strategy with the highest carrying capacity will have the most negative value of the term $\rho_{N} K$, and, on the scale of response, the smallest value of $e^{\rho_{0}+\rho_{N} K}$. In scenario 2 , the fittest strategy will have the most negative value of the term $\beta_{N} K$ and, on the scale of response, the smallest value of $1 /\left(1+e^{-\left(\beta_{0}+\beta_{N} K\right)}\right)$. Evolution consequently acts to minimize the value of the density-dependent rates.

A population at carrying capacity is at an equilibrium size, and the population growth rate $\lambda=1$. As evolution minimizes the value of density-dependent terms, acting to minimize the value of density-dependent demographic rates, other rates must increase, as the population growth rate is a function of per-capita demographic rates. In our models, as we change the development rate, we alter the value of density-independent survival rates: both juvenile and adult survival rates in scenario 1 , and adult survival rate in scenario 2 . The values of the density-independent rates determine the value that the density-dependent rates must take, and consequently carrying capacity.

In many real-world cases, demographic functions include other terms beyond those involving density. For example, in our scenario 2 , where juvenile survival is the density-dependent function, juvenile survival is influenced by body size $\left(\beta_{z}>0\right)$ and density. Population density and mean body size covary across life histories, which complicates the association between juvenile survival rate and fitness $K$ across life histories. Although evolution acts to minimize 
juvenile survival via the density-dependent term $\beta_{N} K$, covariation with mean body size means the least fit strategy does not have the lowest value of juvenile survival, as mean body size is larger in the life history strategy with minimum fitness compared to faster, smaller-bodied life histories. Biologically what this means is evolution can act to maximize one (or more) density-independent terms within a demographic function while simultaneously minimizing another term in the same function. In our scenario 2 , evolution acts to maximize survival via the density-independent term $\beta_{z} z$ while simultaneously minimizing the density-dependent term $\beta_{N} K$.

We include density-dependence in either adult reproduction (scenario 1) or juvenile survival (scenario 2). To gain insights into the dynamics the models predict, it makes sense to write the predicted population dynamics as a function of juvenile and adult age classes and their rates. We do this by writing,

$$
\begin{aligned}
K & =K_{j}+K_{a} \\
K & =N_{j} \bar{S}_{j, K}+N_{a}\left(\bar{S}_{a, K}+\bar{R}_{a, K}\right) \\
1 & =\frac{N_{j}}{K} \bar{S}_{j, K}+\frac{N_{a}}{K}\left(\bar{S}_{a, K}+\bar{R}_{a, K}\right)
\end{aligned}
$$

where $K_{j}$ and $K_{a}$ are the numbers of juveniles and adults at carrying capacity, and $\bar{S}_{x, K}$ and $\bar{R}_{x, K}$ describe mean survival and reproductive rates in age class $x$ at carrying capacity $K$. Reproductive rates will always be zero for juveniles. We refer to the $N_{j} \bar{S}_{j, K}, N_{a} \bar{S}_{a, K}$ and $N_{a} \bar{R}_{a, K}$ as respectively the juvenile survival, adult survival, and adult reproduction terms, and $N_{a}\left(\bar{S}_{a, K}+\bar{R}_{a, K}\right)$ as the adult demographic performance term. We calculate each of these quantities from model predictions using approaches in Coulson et al. (2010). We then explore how these terms vary with size at sexual maturity to gain insights into how inclusion of density-dependence into one demographic rate influences its value and those of the density-independent rates. Note that the equations provide a description of the population dynamics, but are not dynamically sufficient.

We can also usefully summarize the life histories the models describe using survivorship and fertility schedules. In particular, for an age-structured density-dependent life history at carrying capacity we can write the Euler-Lotka identity as

$$
1=\int_{a=0}^{\infty} L(a, K) R(a, K) d a
$$

where $L(a, K)$ and $R(a, K)$ are respectively survivorship to age $a$ and per-capita reproductive success at age $a$, both evaluated at carrying capacity, $K$. Because reproduction does not occur until sexual maturity is reached

$$
1=\int_{a=a_{m}}^{\infty} L(a, K) R(a, K) d a .
$$

where $a_{m}$ is age at sexual maturity. In the models $R(a, K)$ is constant across ages beyond sexual maturity within a 
life history so we simplify to $R(K)$ then write,

$$
1=L\left(a_{m}, K\right) R(K) \frac{\int_{a=a_{m}}^{\infty} L(a, K) d a}{L\left(a_{m}, K\right)} .
$$

${ }_{231}$ The survivorship term $L\left(a_{m}, K\right)$ is the proportion of each cohort surviving to sexual maturity, and $\frac{\int_{a=a_{m}}^{\infty} L(a, K) d a}{L\left(a_{m}, k\right)}$

is life expectancy at sexual maturity that we write as $E\left(a_{m}, K\right)$. This reveals a trade-off between per-capita reproduction, the proportion of each cohort surviving to sexual maturity, and life expectancy at sexual maturity. In scenario $1, R(a, K)$ is density-dependent, so we separate the density-dependent and -independent rates, such that,

$$
\frac{1}{R(a, K)}=L\left(a_{m}\right) E\left(a_{m}\right)
$$

and

$$
-\log (R(a, K))=\log \left(L\left(a_{m}\right) E\left(a_{m}\right)\right) .
$$

We therefore expect to see a trade-off between the product of demographic parameters that are density-independent, with the value of demographic parameters influenced by density-dependence.

We can calculate these continuous age-structured quantities by using Steiner et al.'s (2012) derivation of a stage duration matrix, $\mathbf{P}=(\mathbf{I}-\mathbf{T})^{-1}$ where $\mathbf{I}$ is the identity matrix and $\mathbf{T}=\mathbf{G S}$. Each $i, j$ element in this matrix describes the expected amount of time an individual in stage $i$ will spend in stage $j$ before death. We can sum these elements across columns (rows) to calculate life expectancy for an individual at sexual maturity, and survivorship from the size distribution at birth to the size at sexual maturity (Steiner et al. 2012), providing us with the terms in equation (7).

\section{Results}

\section{Disruptive selection on body size}

In both scenarios we observe disruptive selection on body size (Figure $2(\mathrm{~A}, \mathrm{~B})$ ): for the parameter values we work with we observe a life history strategy of minimum fitness. To the left of this threshold on the $x$-axis we see directional selection for small body size at sexual maturity and a fast life history, while to the right of it the opposite pattern is observed.

\section{Fitness Maximization}

Carrying capacity is fitness in negative density-dependent integral projection models (Kentie et al. 2020), with evolution consequently acting to maximize $K$. As carrying capacity increases across life histories, intercepts plus 
terms that include $K$ in the functions will decrease in value on the scale of response (see above). As an example, in scenario 1 , the value of the term $e^{\rho_{0}+\rho_{N} N}$ in the reproduction function changes across life history. However, the survival and reproduction functions can also include terms describing the effect of body size on the demographic rate. For example, in scenario 2, juvenile survival is influenced by both density and body size. We deliberately formulated the two scenarios, by setting some parameters to zero (Table 2), so that models contain only one density-dependent term, and only one body size term across the survival and reproduction functions. In scenario one, density influences reproduction while body size influences survival, while in scenario two, reproduction is constant and juvenile survival is influenced by body size and population density, and adult survival is influenced by body size.

As we change the development function (and asymptotic size and size at sexual maturity), both mean survival rates and survivorship schedules also change even though the size-survival function remains constant. The reason for this, illustrated below, is because individuals are developing at a faster rate if they achieve larger body sizes at sexual maturity. As survival and survivorship schedules change, the density-dependent term in the density-dependent demographic rate function must change in response to achieve a value such that $\lambda=R 0=1$ resulting in a stable size-structure (Figure 2(C,D)).

The life history and population dynamics this (relatively) simple feedback generates are surprisingly nuanced. We consider each of the two scenarios in turn.

\section{Population Dynamics}

We were unable to identify life history trade-offs by examining per-time step juvenile survival, adult survival, and adult reproductive, and instead observed very different patterns across the two scenarios. We show this by drawing on results from the two scenarios.

In scenario 1, the fittest strategies tended to have a lower proportion of juveniles (Figure $\mathrm{S} 1(\mathrm{~A})$ ) and a higher proportion of adults (Figure S1(B)) in the population compared to less fit strategies. As life histories evolve to be faster, with smaller body sizes, we observe negative covariances between both juvenile (Figure S1(C)) and adult survival (Figure S1(D)) and the adult reproductive rate, with positive covariances observed in the parameter space where large bodied sized, slower life histories evolve. As expected, evolution minimized adult per-capita reproduction, the density-dependent rate (Figure S1(E). There was a positive covariance between juvenile and adult survival rates across life histories (Figure S1(F)), with positive covariances between both survival rates and reproduction in the regime of parameter space where fast life histories evolve, and negative covariances in the regime where slower life histories were selected (Figures $\mathrm{S} 1(\mathrm{G}, \mathrm{H})$ ).

In scenario 2, in the parameter space where slower, larger-bodied life histories were selected, fitter strategies tended to have a greater proportion of juveniles in the population (Figure S2(A)). In contrast, when fast life histories were selected, the fitter strategies tended to have a high proportion of adults in the population (Figure S2(B)). These 
patterns stem from our general observation of evolution minimizing density-dependent terms while maximizing density-independent ones. The association between juvenile survival and carrying capacity was highly non-linear (Figure S2(C)). When fast life histories were selected, adult survival rates negatively covaried with carrying capacity (Figure S2(D)), while the opposite was observed when slower life histories were selected. Because reproductive rate was a constant, it did not covary with carrying capacity (Figure S2(E)), or other rates (Figure S2(G,H). There was a highly non-linear association between juvenile and adult survival rates (Figure S2(F)).

\section{Life History Dynamics for Scenario 1, Density-dependent Reproduction}

Although exploration of the per-time step demographic rates did not allow us to gain general insight into where life history trade-offs underpinning selection on body size lie, analysis of the survivorship schedules does. Holding the size-survival function constant (Figure 1(D)), but altering the development function (Figure 1(B,C)), inevitably changes the survivorship functions: the probability of surviving from birth to a particular age (Figure 3(A)). The faster that individuals grow, the more quickly they progress along the $x$-axis of the body size-survival function (Figure 1(D)), and this means that their probability of surviving at each age increases when going from fast-lived to slow-lived life histories.

The change in the development function, and in size and age at sexual maturity, generates variation in the probability of an individual surviving to sexual maturity across life histories (Figure 3(B)). A smaller proportion of each cohort achieves sexual maturity as size at sexual maturity increases because it takes longer to achieve sexual maturity, and this delay imposes a greater mortality burden on each cohort than the survival benefits accrued via achieving larger sizes at a particular juvenile age (Figure $1(\mathrm{C})$ ). The mortality cost of delaying sexual maturity can be offset by an increase in life expectancy at sexual maturity (Figure 3(C)) as larger adults have higher per-time step survival rates than those that are smaller (Figure 3(D)) and consequently live for longer.

Below a threshold (green line in Figure 3(B-E)) the proportion of the population achieving sexual maturity decreases at a relatively faster rate than the corresponding increase in life expectancy, with the converse true above the threshold. A consequence of these contrasting rates of change is that the proportion of sexually mature individuals within the population can increase (Figure 3(D)), even though a smaller proportion of each cohort achieves sexual maturity (Figure 3(B)), simply because a greater number of cohorts are alive as adults at any one time as adult life expectancy increases. Once individuals achieve sexual maturity, they reproduce.

The switch in the relative sizes of the derivative of the proportion of each cohort surviving to sexual maturity to size at sexual maturity, and the derivative of life expectancy to size at sexual maturity, generates disruptive selection. We observe an "n"-shaped association between size at sexual maturity and the per-capita reproductive rate (Figure 3(E)), which is reflected in a mirror-image " $\mathrm{u}$ "-shaped association between size at sexual maturity and carrying capacity (Figure $2(\mathrm{~A})$ ). The constraint $R 0=1$ means that the minimization of the density-dependent 
term in the density-dependent reproduction function must be countered by maximization of values predicted by the density-independent body size term in the survivorship function. Because the survivorship function determines both the proportion of each cohort that achieves sexual maturity, and life expectancy at sexual maturity, and given equation (8), we observe a linear association with a slope of -1 between the log of the product of survivorship to sexual maturity and life expectancy at sexual maturity with the log of the per-capita reproductive rate (Figure 3(F)).

We can now understand why we observe disruptive selection on size at sexual maturity (and life history speed). Below the threshold of minimum fitness (vertical green dotted lines in Figure 3), the density-independent body size term in the survival function is maximized by maximizing the proportion of each cohort that survives to sexual maturity. This is achieved by selecting for an ever-earlier size at sexual maturity to minimize the amount of mortality between birth and sexual maturity. In contrast, above this threshold, the density-independent survival term is maximized by maximizing life expectancy at sexual maturity, and this is achieved by selecting for an increase in size at sexual maturity.

In Figure 4 we illustrate how density-dependent and density-independent functions can change with age as described above to ensure the constraint $R 0=\int_{a=0}^{\infty} L(a, K) R(a, K) d a=1$ and impose selection on size at sexual maturity. The summary figure does not include body size because its inclusion complicates visual interpretation (Figure S3). The figure shows how a change in age at sexual maturity (4(A) versus $4(\mathrm{~B}))$ results in a change in the form of the survivorship function, which results in a change in the elevation of the density-dependent reproductive function to ensure $R 0=1$. The life history in Figure 4(B) is favoured by selection in this example because the density-dependent reproductive function is at a lower elevation than in Figure 4(A). Figure 4(C) provides an explanation of the rectangle approximation used in equation (6).

\section{Life History Dynamics for Scenario 2, Density-dependent Juvenile Survival}

We now consider the case where juvenile survival is density-dependent; in contrast to the reproduction function, survival is also dependent on body size. Reproduction is now density-independent and $\rho_{N}=0$. A consequence of these changes is the form of the survival and survivorship functions now differ compared with scenario 1 (compare Figures 5(A, B) with Figures 2(D) and 3(A)). The density-independent rate that is now maximized is adult survival. Per-capita reproduction cannot be maximized as it does not vary with life history (because $\rho_{z}=0$ and $\rho_{N}=0$ ).

As before, the proportion of each cohort achieving sexual maturity declines with increasing size at sexual maturity (Figure 5(C)), while life expectancy increases (Figure 5(D)). These processes combine to generate a quadratic association between size at sexual maturity and the proportion of the population that is sexually mature (Figure $5(\mathrm{E}))$. The same maximization of $K$, and minimization of the density-dependent term occurs as in scenario 1 , except the demographic rate that is modified is now $S\left(z<z_{a}, N, t\right)$ (rather than $R(z, N, t)$ ), and the term being minimized is now $\beta_{0}+\beta_{N} K$ (rather than $\rho_{0}+\rho_{N} K$ ). The density-independent life history quantity that is now maximized is 
adult life expectancy (Figure 5(D)).

There is one significant difference compared to scenario 1: survival $\left(\beta_{z}>0\right)$, unlike reproduction $\left(\rho_{z}=0\right)$, is a function of body size. Because the development function varies across life histories along with size at sexual maturity, mean juvenile body size, and consequently mean juvenile survival, also varies with life history. A consequence of juvenile survival being influenced by both density and body size, and mean juvenile body size varying across life histories, is that the life history with minimum fitness does not align with the life history that has the maximum per-capita juvenile survival rate (Figure $5(\mathrm{~F})$ ). This does not affect the negative linear association between the logs of the density-independent and density-dependent rates (Figure $5(\mathrm{G})$ ).

\section{Changing the survival function}

The reason why the proportion of each cohort that achieves sexual maturity and life expectancy at sexual maturity change at different rates across life histories in the models, generating disruptive selection on size at sexual maturity, is the non-linear form of the size-survival function, and the rate at which survival changes with size (and age). The elevation and slope of the size-survival function should consequently determine the shape of selection on life history. We examined this for scenario 1 by systematically modifying the two parameters (Figure 6).

When the slope of the body-size survival function is 0 we never observe selection for delayed age and size at sexual maturity and a slower life history (column 1). In order to see selection for an increase in size at sexual maturity, survival rates need to increase with body size (positive viability selection) and need to be sufficiently high for sexually mature adults to extend lifespan sufficiently to offset the costs of a smaller proportion of offspring surviving to sexual maturity (see equation (6)). It is this fitness differential across ages that determines whether there will be selection for an increase or decrease in body size and age at sexual maturity.

We also investigated whether our results remained when we linearised approxated the model (Appendix 1). They did, revealing that the patterns are not a consequence of the weak non-linearities in our simulations.

\section{Discussion}

We explore circumstances under which extremes of body size and life history speed are expected to evolve. We assumed a density-dependent environment, because, over evolutionary time, populations do not show persistent, long-term temporal trends in numbers (Coulson 2021). Despite the simplicity of the models we develop, the results reveal several novel insights. 


\section{Why do extremes of body size evolve?}

Although our models have only one function being density-dependent, and the only way for evolution to maximize density-independent functions is via modification of the developmental trajectories, we found areas of parameter space where small-bodied, fast life histories evolved and other areas where the converse was true. Our results reveal that if delaying age at sexual maturity also increases size at sexual maturity, and this increases the mortality rate between birth and sexual maturity, then we would expect smaller-bodied, fast life histories to evolve unless this increased juvenile fitness cost was offset by an increase in adult fitness (see also Stearns \& Koella (1986)). In the models we develop, the increase in adult fitness came via an extension to adult lifespan. However, it could also come via an increase in per-capita per-time step reproductive output (Kozłowski et al. 2020), which could occur if reproduction increased with body size as is observed in some species (Barneche et al. 2018). Although negative density-dependence can operate in any demographic rate (Eberhardt 2002), we focused on reproduction and juvenile survival as this is where it appears to operate most frequently (Bonenfant et al. 2009).

The simple models we develop predict runaway disruptive selection - in other words, all species should evolve to be either dwarfs or giants. This does not happen, so some process must prevent such runaway selection. At the extremes, it seems plausible that physiological or metabolic constraints impose maximum and minimum sizes (West et al. 1997), but what prevents runaway selection in animals of intermediate size? We show that once survival rates plateau with size, then selection for an increase in size at sexual maturity ceases. In other words, once survival rates plateau, there are no longer adult fitness gains available via delaying sexual maturity to extend life expectancy.

One process that results in survival decreasing with age is senescence. It is important to note that we do not incorporate senescence into models, but given its ubiquitous nature (Nussey et al. 2013), it seems plausible that senescence means that survival rates cannot remain indefinitely high among adults. Depending on the age at which senescence begins, and how quickly it happens, there could be a trade-off between rates of development, the shape of the size-survivorship function, and the onset of senescence (Jones et al. 2008). Future work should incorporate the effects of both age and body size on survival and reproduction (and offspring size (Charnov \& Downhower 1995)) into models to explore the conditions under which runaway selection for ever-smaller, or -larger, body size can be constrained.

\section{Minimization, Maximization and the Emergence of Trade-offs}

The minimization of some demographic terms and the maximization of others in the models we develop generate within- and between-age trade-offs. These are evident in the life history rates, but are not apparent in the per-time step demographic rates used to describe the population dynamics. We start by considering the life history rates.

In scenario 1, where the density-dependent feedback operates via reproduction, the minimization of terms in the reproductive function results in either maximization of juvenile or adult survival rates via the survivorship functions 
depending on whether the relative rate of change in the proportion of each cohort achieving sexual maturity, or life expectancy at sexual maturity, changes fastest with size at sexual maturity. Put another way, the absolute values of the sensitivities of the proportion of each cohort achieving sexual maturity to size at sexual maturity, and life expectancy to size at sexual maturity, determine the direction of selection. We can consequently observe negative covariances (trade-offs) between juvenile survivorship and adult reproduction, but also between adult life expectancy and adult reproduction.

These trade-offs are most easily understood via examination of the changing shapes of the survivorship and reproductive schedules (Stearns 1977) rather than via the population dynamic rates. The main reason for this is we can describe life histories with three rates - the proportion of each cohort surviving to sexual maturity (which is equal to survivorship to sexual maturity), life expectancy at sexual maturity, and the adult per-capita reproductive rate. With only three terms to work with, it is (relatively) straightforward to identify trade-offs. In contrast, the description of the population dynamics we use involves five terms - the proportion of juveniles in the population, the proportion of adults in the population, the per-time step juvenile survival rate, the per-time step adult survival rate, and the adult per-capita reproductive rate. Because these change in different ways and at different rates with size at sexual maturity, identifying trade-offs by examining negative covariances between pairs of rates becomes impossible. This result highlights the challenge of identifying trade-offs from demographic rate data underpinning population dynamics (Tavecchia et al. 2005).

The life history description we use in terms of survivorship and reproductive schedules does not explicitly include information on the population structure, and this simplifies the challenge of identifying the trade-offs that emerge as we only work with three terms. At one level the approach we develop is general, as all life histories can be divided into juvenile and adult age-classes as a function of whether individuals are sexually mature or not. By setting the reproductive rate as a constant in adults, rather than having it vary with body size as it does in many species (Barneche et al. 2018), we could easily reformulate the Euler-Lotka relationship in a way that allows us to summarize the adult survivorship schedule as life expectancy - the average of this schedule. It should always be possible to rectangularize the product of the survivorship and fertility schedules post-sexual maturity into life expectancy and average per-time adult reproductive rate given survivorship to sexual maturity, so the approach is general. However, a more nuanced understanding, particularly when senescence is included, would usefully involve sensitivities of life expectancy and adult reproductive output to survivorship and reproductive output at each age and size given size at sexual maturity (Tuljapurkar et al. 2009; Steiner et al. 2012; Jones \& Tuljapurkar 2015).

Age-class-specific survival rates are determined by the shape of the size-survival function and the development function (Tuljapurkar et al. 2009). In many species, per-time step survival rates increase with body size (Ronget et al. 2018), so the functional form we chose for these functions is appropriate. However, how these functions translate into survivorship functions depends upon the form of the development functions. If the development function 
was density-dependent, such that the rate of development decreased with density, then as density increased, the survivorship curves will change, even for a constant size-survival function, simply because individuals will develop more slowly. All other things being equal, this would be expected to select for a smaller-sized, faster life history, as a smaller proportion of each cohort would survive to sexual maturity. Development rates consequently indirectly determine the life history by determining how quickly survival rates change with age. Development rates vary with age as a function of the environment in many species, and are described by reaction norms (Stearns \& Koella 1986; Murren et al. 2014). When density is low, and resources are abundant, or at ideal temperature conditions in ectotherms, body size can develop quickly, while rates are lower when food is scarce or temperature is away from the optima (Day \& Rowe 2002). One key question that arises is whether the size-survival function will also change as environmental variables change? In many species, it appears to do so, potentially because rates of development of traits other than body size are also influenced by the environment (Gaillard et al. 2000). Understanding covariation between phenotypic trait-survival function and development rate functions would allow us to extend the approach to cases where we do not assume a constant survival function across life histories.

We kept offspring size constant in models. However, changing offspring size could also impact life history evolution (Winkler \& Wallin 1987; Reznick et al. 1990; Charnov \& Downhower 1995). First, if carrying capacity measured in total number of individuals is fitness, and density-dependence operates via reproduction, then reducing litter size while increasing offspring size is one route to evolving a lower per-capita reproductive rate allowing persistence at a higher carrying capacity. Second, larger offspring begin life further along the body size-survival function, potentially increasing the proportion of each cohort that survives to sexual maturity, altering the strength of selection on size at sexual maturity and life history speed. As we increase the degrees of freedom via which survivorship and reproductive schedules can be altered, we reduce the constraints on how species may respond to environmental change. Nonetheless, working in situations where carrying capacity is fitness imposes a constraint that will generate trade-offs, and the approach we develop provides a way to identifying the types of trade-offs that might emerge as a function of the part of the life history where density-dependence operates most strongly.

\section{Carrying Capacity as Fitness}

Density-dependence can be generated by any limiting resource, and this means we can interpret fitness as being determined by a number of different limiting factors. In this section we consider the biological interpretation of carrying capacity as fitness.

The mechanism via which density-dependence impacts life history evolution in the models we develop is to minimize density-dependent terms in demographic functions when evaluated at $K$. If per-capita reproduction in strategies $A$ and $B$ is influenced in the same manner by density, the life history strategy that can persist with the lowest per-capita reproductive rate for any given value of $z$ is the fittest. Evolution acts to minimize the sensitivity of the 
demographic rate to population density.

Carrying capacity is often defined as the total number of individuals a particular environment can support (Turchin 1999). However, it can be specified in other ways. What if total biomass, e.g. $\int_{0}^{\infty} z N(z, K, t) d z$, is a better descriptor of the density-dependent feedback (Owen-Smith 2002) than just total population size at equilibrium? Fitness is now total biomass, and evolution will maximize biomass and will minimize the value of the terms in demographic functions that include biomass. We can extend this logic further to cases where individuals with different values of a phenotypic trait, such as body size, impose different competitive pressures on one another (Bolker et al. 2003). $K$ can now be defined as the sum of the product of a pairwise trait-mediated interaction surface with the density distribution of conspecifics with each trait value (Bassar et al. 2016). The pairwise interaction surface describes the competitive impact of an individual with trait value $z_{i}$ on an individual with trait value $z_{j}$ across all values of $i, j$, scaled such that two individuals with the same value of the trait impose a competitive pressure on one another of unity (McCoy \& Bolker 2008). The life history strategy that can persist at the highest value of the term describing this more complex negative feedback will be the fittest (Bassar et al. 2016). $K$ is consequently defined here as being more nuanced than the total number of individuals in a population at equilibrium.

Density-dependence (and consequently carrying capacity) is used as a shortcut to summarize intraspecific competitive interactions for a shared resource, trophic interactions such as predation or herbivory that lead to competition, or indirect interspecific competitive interactions (Hassell 1986). A consequence of this is we can replace density feedbacks with other terms, such that carrying capacity can become a function of the number, and/or population structure, of other species (Bagchi et al. 2010). For example, the trait-mediated interaction surface and the density distribution of trait values among conspecific competitors described above can be modified to capture interspecific competition. In communities of indirectly competing species on the same trophic level, the strength of species interactions can be characterized with interaction coefficients describing the competitive impact of one individual of species $A$ on an individual of species $B$ (Hofbauer et al. 1987; Allesina \& Tang 2012). A species interaction matrix, similar to the trait interaction surface, and the distribution of individuals in each competing species can now be used to calculate a quantity that is an interspecific definition of $K$. If the phenotypic trait structure of an interacting species influences the outcome of interspecific interactions, then this too could be incorporated into models via a more complex definition of $K$ (Bassar et al. 2017). This naturally allows the effect of different competitive environments on life history evolution to be explored.

Interspecific competition describes indirect interactions via a shared resource, but we can also define $K$ for directly interacting species. When we know what the limiting process is, we can replace carrying capacity with an expression describing the strength of the trophic interaction (Adler et al. 2010). For example, in a predator-limited population we might now write an equation for a demographic rate influenced by the equilibrium number of predators $P$ as $V(z, P)=\nu_{0}+\nu_{z} z+\nu_{P} P$ where $\nu_{P}<0$. Evolution will now maximize the value of $P$, minimizing the value of 
$V(z, P)$. Biologically this means that in a predation-limited environment evolution is selecting for life histories that can persist at the highest predator densities. We could now construct a dynamic model of a focal prey and its predator population, and examine how a life history evolves in response to changing the way predation operates.

The effect of the number of predators on a focal population is determined by a negative parameter (in the example above, $\left.\nu_{P}\right)$. In a food-limited population, where $\Phi$ is the equilibrium availability of food, we might write $V(z, \Phi)=\omega_{0}+\omega_{z} z+\omega_{\Phi} \Phi$ where $\omega_{\Phi}>0$. In this case, evolution will act to minimize food availability $\Phi$, maximizing the value of the function $V(z, \Phi)$. The fittest life history strategy is now the one that can survive on the least amount of food, with the resulting dynamic being equivalent to Tilman's $R *$ concept (Tilman 1982). Energy availability could be substituted for food, allowing models to be extended to examine circumstances when metabolic scaling rules might emerge.

These insights into the definition of fitness are important as we can now define fitness for dynamic models of directly and indirectly interacting species at equilibrium, with fitness being equal to the population size and/or structure (or biomass) of the species directly imposing the feedback. This aids understanding of how species are expected to adapt to environments via phenotypic trait evolution. For example, in environments where predation determines the feedback, evolution will select traits that enable prey to live with predators (Coulson 2021). Such traits might be camouflaged coloration, social living, anti-predator behaviors, and morphological characteristics that aid escape from predators (Reznick \& Endler 1982).

In the discussion to date (and in our models), we have assumed each life history strategy must be influenced by the same fitness metric, $K$, whether it is calculated as a function of the distribution of intraspecific competitors, interspecific competitors, or trophically interacting species, or even a mixture. What if each life history strategy has a different definition of $K$ ? Under this scenario, we can draw on insight from modern coexistence theory, and the role of fitness differences on coexistence (Chesson 2000). When fitness is defined differently for two different life history strategies, they will exhibit fitness differences that arise from niche differentiation, and this provides a pathway to coexistence. Appreciating that in some circumstances fitness can be defined as a property of interacting species opens the possibility of more formal links between ecology, which aims to understand species interactions, and evolution, which is concerned with within-population change.

\section{Empirical Considerations}

Our work is theoretical, but it leads to a number of hypotheses that could be empirically tested. We show that the shapes of the four function types used to construct models determine whether small-bodied and fast, or large-bodied and slow, life histories are selected. To understand why a particular body size and life history evolves, it is consequently insightful to explore why the survival, development, reproduction, and inheritance functions take the shapes they do, and how they covary. What are the genetic, physiological, or environmental factors that determine 
the size-survival function, for example (Coulson 2021)? As a population adapts to a new environment, the strength and form of feedbacks may change, and this will be reflected in the way the functions that constitute models change as adaptation occurs. Not only will this help us understand phenotypic trait and life history evolution, but also the way that the population dynamics change as adaptation occurs as these are easily studied using IPMs. Understanding why we see particular functional forms, and how these change as adaptation progresses will provide novel insight, but the approach also has the potential to help explain a number of evolutionary "rules".

There are three main biogeograpical "rules' describing patterns of body size: the island rule, Bergmann's rule, and Cope's rule. The island rule states that small species of many mammals and birds tend to evolve large body sizes and slower life histories on islands, while larger species tend to evolve in the other direction (Clegg \& Owens 2002; Lomolino 2005; Covas 2012; Sandvig et al. 2019). Bergmann's rule states that an increase in latitude typically corresponds to an increase in adult body sizes within species (McNab 1971). Cope's rule states that species tend to get larger over evolutionary time (Hone \& Benton 2005), suggesting a similar process could well be happening over time as happens with latitude. These patterns suggest systematic changes in the shapes of size-survival, size-reproduction, development rates, and offspring size may underpin these"rules". Additional work, where we impose fewer constraints on the functions in models, should help explain the circumstances required to generate these body size and life history patterns.

We can even hypothesize on the shape of the functions in extinct species, such as the giant sauropods. These giants are thought to have laid multiple clutches of relatively few ostrich egg-sized eggs, have very high early growth rates, and to achieve sexual maturity at around 50-70 years (Sander et al. 2011). The high growth rates suggested the young were unlikely food-limited, and selection for very large size suggests a steep increase in survival rates across the range of sizes through which they developed. Taken together, these suggest a high mortality rate on the young, likely via predation, but long life expectancies once sexual maturity was achieved.

\section{Limitations and Next Steps}

One obvious limitation of the models we develop is they do not include environmental stochasticity. One effect of environmental stochasticity is to periodically reduce population size such that feedbacks do not strongly operate. For example, in a model with density-dependent feedback, environmental stochasticity may result in periodic declines in population size when the feedback has little impact on the demographic rates via which it operates. The population will now undergo a period of rapid, stochastic growth, until the feedback starts to operate again to limit population size (Lande et al. 2009). During the period when the feedback is not operating, trade-offs will be negligible as no rates are being minimized, and the fittest life history strategy will be the one that can maximize the most demographic rates, and will consequently always be the one with the highest stochastic growth rate (Tuljapurkar 1990). This will always select for a fast life history with a small body size. In a stochastic environment with 
feedbacks, and a stationary distribution of population size, the fittest life history strategy will consequently depend upon the proportions of time the population is subject to the feedback operating strongly, and the proportion of time they are largely absent (Schreiber 2021). More specifically, there will exist a frequency distribution of the strength of the feedback, and this will determine the evolutionarily stable life history strategy in a stochastic environment with feedbacks. There has been significant work incorporating stochasticity into modern coexistence theory, and this could help with extending our approach to stationary stochastic environments.

\section{Conclusions}

There are many ways in which the models we use can be extended and parameterized to address a range of empirical and theoretical questions about body size and life history evolution. Our work also contributes to a general framework that we have been developing where we consider natural systems as attaining quasi-stationary states from which they can be perturbed before starting an eco-evolutionary journey to a new stationary state (Coulson et al. 2011, 2017). The journey can be characterized with dynamical change in the form of the fundamental demographic functions used to construct models. Work to date with integral projection models has primarily focused on the dynamics of phenotypic traits (Coulson et al. 2010), genes (Coulson et al. 2011), and population (Ellner et al. 2016) and community dynamics (Adler et al. 2010). The work we report here shows how life history evolution can be examined using models within the framework we are developing, and insights extended to modern coexistence theory, and trophic interactions. Integral Projection Models provide a remarkably powerful suite of tools to address a vast array of questions in population and community ecology, life history and quantitative trait evolution (Ellner et al. 2016). The next empirical set of questions we will explore will examine whether this class of model performs as well in shedding light on life history evolution as they have on ecological dynamics and phenotypic trait evolution.

\section{Acknowledgements}

Thanks to Luke Coulson for running simulations over a range of parameter values. Thanks to Mike Furlong and the School of Biological Sciences at the University of Queensland for hosting TC's and SC's sabbaticals where the work was largely conducted. RDB is supported by NSF DEB2100163; Travis is supported by National Science Foundation award DEB 2100163; GP is funded by the UK's Natural Environment Research Council through the Doctoral Training Partnership in Environmental Research at the University of Oxford (NE/L002612/1);

\section{References}

Adler, P.B., Ellner, S.P. \& Levine, J.M. (2010). Coexistence of perennial plants: An embarrassment of niches. Ecol. Lett., 13, 1019-1029.

Allesina, S. \& Tang, S. (2012). Stability criteria for complex ecosystems. Nature, 483, 205-208. 
Audzijonyte, A., Richards, S.A., Stuart-Smith, R.D., Pecl, G., Edgar, G.J., Barrett, N.S., et al. (2020). Fish body sizes change with temperature but not all species shrink with warming. Nat. Ecol. Evol., 4, 809-814.

Bagchi, R., Swinfield, T., Gallery, R.E., Lewis, O.T., Gripenberg, S., Narayan, L., et al. (2010). Testing the Janzen-Connell mechanism: Pathogens cause overcompensating density dependence in a tropical tree. Ecol. Lett., $13,1262-1269$.

Barneche, D.R., Robertson, D.R., White, C.R. \& Marshall, D.J. (2018). Fish reproductive-energy output increases disproportionately with body size. Science, $360,642-645$.

Bassar, R.D., Childs, D.Z., Rees, M., Tuljapurkar, S., Reznick, D.N. \& Coulson, T. (2016). The effects of asymmetric competition on the life history of trinidadian guppies. Ecol. Lett., 19, 268-278.

Bassar, R.D., Travis, J. \& Coulson, T. (2017). Predicting coexistence in species with continuous ontogenetic niche shifts and competitive asymmetry. Ecology, 98, 2823-2836.

Bolker, B., Holyoak, M., Křivan, V., Rowe, L. \& Schmitz, O. (2003). Connecting theoretical and empirical studies of trait-mediated interactions. Ecology, 84, 1101-1114.

Bonenfant, C., Gaillard, J.-M., Coulson, T., Festa-Bianchet, M., Loison, A., Garel, M., et al. (2009). Empirical evidence of density-dependence in populations of large herbivores. Adv. Ecol. Res., 41, 313-357.

Caswell, H. (2001). Matrix population models: Construction, analysis, and interpretation. Sinauer Associates.

Charlesworth, B. (1994). Evolution in age-structured populations. Cambridge University Press Cambridge.

Charnov, E.L. \& Downhower, J.F. (1995). A trade-off-invariant life-history rule for optimal offspring size. Nature, $376,418-419$.

Chesson, P. (2000). Mechanisms of maintenance of species diversity. Annu. Rev. Ecol. Syst., 31, 343-366.

Childs, D.Z., Rees, M., Rose, K.E., Grubb, P.J. \& Ellner, S.P. (2004). Evolution of size-dependent flowering in a variable environment: Construction and analysis of a stochastic integral projection model. Proc. Roy. Soc. B., $271,425-434$.

Clegg, S.M. \& Owens, P. (2002). The 'island rule'in birds: Medium body size and its ecological explanation. Proc. Roy. Soc. B., 269, 1359-1365.

Coulson, T. (2012). Integral projections models, their construction and use in posing hypotheses in ecology. Oikos, $121,1337-1350$.

Coulson, T. (2021). Environmental perturbations and transitions between ecological and evolutionary equilibria: An eco-evolutionary feedback framework. Peer Community Journal, 1.

Coulson, T., Benton, T., Lundberg, P., Dall, S., Kendall, B. \& Gaillard, J.-M. (2006). Estimating individual contributions to population growth: Evolutionary fitness in ecological time. Proc. Roy. Soc. B., 273, 547-555.

Coulson, T., Kendall, B.E., Barthold, J., Plard, F., Schindler, S., Ozgul, A., et al. (2017). Modeling adaptive and nonadaptive responses of populations to environmental change. Am. Nat., 190, 313-336.

Coulson, T., MacNulty, D.R., Stahler, D.R., VonHoldt, B., Wayne, R.K. \& Smith, D.W. (2011). Modeling effects of 
environmental change on wolf population dynamics, trait evolution, and life history. Science, 334, 1275-1278.

Coulson, T., Tuljapurkar, S. \& Childs, D.Z. (2010). Using evolutionary demography to link life history theory, quantitative genetics and population ecology. J. Anim. Ecol., 79, 1226-1240.

Covas, R. (2012). Evolution of reproductive life histories in island birds worldwide. Proc. Roy. Soc. B., 279, $1531-1537$.

Day, T. \& Rowe, L. (2002). Developmental thresholds and the evolution of reaction norms for age and size at life-history transitions. Am. Nat., 159, 338-350.

Day, T. \& Taylor, P.D. (1997). Von Bertalanffy's growth equation should not be used to model age and size at maturity. Am. Nat., 149, 381-393.

Depczynski, M. \& Bellwood, D.R. (2005). Shortest recorded vertebrate lifespan found in a coral reef fish. Curr. Biol., 15, R288-R289.

Dieckmann, U., Heino, M. \& Parvinen, K. (2006). The adaptive dynamics of function-valued traits. J. Theor. Biol., 241, 370-389.

Eberhardt, L. (2002). A paradigm for population analysis of long-lived vertebrates. Ecology, 83, 2841-2854.

Ellner, S.P., Childs, D.Z. \& Rees, M. (2016). Data-driven modelling of structured populations: A practical guide to the integral projection model. Springer.

English, S., Bateman, A.W. \& Clutton-Brock, T.H. (2012). Lifetime growth in wild meerkats: Incorporating life history and environmental factors into a standard growth model. Oecologia, 169, 143-153.

Fisher, S., Ronald Aylmer. (1930). The genetical theory of natural selection. Clarendon Press, Oxford.

Gaillard, J.-M., Festa-Bianchet, M., Yoccoz, N., Loison, A. \& Toigo, C. (2000). Temporal variation in fitness components and population dynamics of large herbivores. Annu. Rev. Ecol. Syst., 31, 367-393.

Gaillard, J.-M., Pontier, D., Allaine, D., Loison, A., Herve, J.-C. \& Heizman, A. (1997). Variation in growth form and precocity at birth in eutherian mammals. Proc. Roy. Soc. B., 264, 859-868.

Grafen, A. (1999). Formal darwinism, the individual-as-maximizing-agent analogy and bet-hedging. Proc. Roy. Soc. B., 266, 799-803.

Grant, A. (1997). Selection pressures on vital rates in density-dependent populations. Proc. Roy. Soc. B., 264, 303-306.

Hassell, M.P. (1986). Detecting density dependence. Trends Ecol. Evol., 1, 90-93.

Hofbauer, J., Hutson, V. \& Jansen, W. (1987). Coexistence for systems governed by difference equations of lotka-volterra type. J. Math. Biol., 25, 553-570.

Hone, D.W. \& Benton, M.J. (2005). The evolution of large size: How does Cope's Rule work? Trends Ecol. Evol., $20,4-6$.

Jones, J.H. \& Tuljapurkar, S. (2015). Measuring selective constraint on fertility in human life histories. P. Natl. Acad. Sci. USA., 112, 8982-8986. 
Jones, O.R., Gaillard, J.-M., Tuljapurkar, S., Alho, J.S., Armitage, K.B., Becker, P.H., et al. (2008). Senescence rates are determined by ranking on the fast-slow life-history continuum. Ecol. Lett., 11, 664-673.

Kentie, R., Clegg, S.M., Tuljapurkar, S., Gaillard, J.-M. \& Coulson, T. (2020). Life-history strategy varies with the strength of competition in a food-limited ungulate population. Ecol. Lett., 23, 811-820.

Kingsolver, J.G., Hoekstra, H.E., Hoekstra, J.M., Berrigan, D., Vignieri, S.N., Hill, C., et al. (2001). The strength of phenotypic selection in natural populations. Am. Nat., 157, 245-261.

Kooijman, B. \& Kooijman, S. (2010). Dynamic energy budget theory for metabolic organisation. Cambridge University Press.

Koziowski, J. \& Weiner, J. (1997). Interspecific allometries are by-products of body size optimization. Am. Nat., $149,352-380$.

Kozłowski, J. (1992). Optimal allocation of resources to growth and reproduction: Implications for age and size at maturity. Trends Ecol. Evol., 7, 15-19.

Kozłowski, J., Konarzewski, M. \& Czarnoleski, M. (2020). Coevolution of body size and metabolic rate in vertebrates: A life-history perspective. Biol. Rev., 95, 1393-1417.

Lachish, S., Brandell, E.E., Craft, M.E., Dobson, A.P., Hudson, P.J., MacNulty, D.R., et al. (2020). Investigating the dynamics of elk population size and body mass in a seasonal environment using a mechanistic integral projection model. Am. Nat., 196, E23-E45.

Lande, R., Engen, S. \& Saether, B.-E. (2009). An evolutionary maximum principle for density-dependent population dynamics in a fluctuating environment. Philos. T. Roy. Soc. B., 364, 1511-1518.

Law, R. (1979). Optimal life histories under age-specific predation. Am. Nat., 114, 399-417.

Lomolino, M.V. (2005). Body size evolution in insular vertebrates: Generality of the island rule. J. Biogeogr., 32, 1683-1699.

McCoy, M.W. \& Bolker, B.M. (2008). Trait-mediated interactions: Influence of prey size, density and experience. J. Anim. Ecol., 478-486.

McGraw, J.B. \& Caswell, H. (1996). Estimation of individual fitness from life-history data. Am. Nat., 147, 47-64.

McNab, B.K. (1971). On the ecological significance of Bergmann's Rule. Ecology, 52, 845-854.

Merilä, J., Sheldon, B. \& Kruuk, L. (2001). Explaining stasis: Microevolutionary studies in natural populations. Genetica, 112, 199-222.

Meszena, G., Kisdi, E., Dieckmann, U., Geritz, S.A. \& Metz, J.A. (2002). Evolutionary optimisation models and matrix games in the unified perspective of adaptive dynamics. Selection, 2, 193-220.

Metcalf, C., Rose, K., Childs, D., Sheppard, A., Grubb, P. \& Rees, M. (2008). Evolution of flowering decisions in a stochastic, density-dependent environment. P. Natl. Acad. Sci. USA., 105, 10466-10470.

Murren, C.J., Maclean, H.J., Diamond, S.E., Steiner, U.K., Heskel, M.A., Handelsman, C.A., et al. (2014). Evolutionary change in continuous reaction norms. Am. Nat., 183, 453-467. 
Nielsen, J., Hedeholm, R.B., Heinemeier, J., Bushnell, P.G., Christiansen, J.S., Olsen, J., et al. (2016). Eye lens radiocarbon reveals centuries of longevity in the greenland shark (somniosus microcephalus). Science, 353, 702-704.

Nussey, D.H., Froy, H., Lemaitre, J.-F., Gaillard, J.-M. \& Austad, S.N. (2013). Senescence in natural populations of animals: Widespread evidence and its implications for bio-gerontology. Ageing Res. Rev., 12, 214-225.

Owen-Smith, N. (2002). A metaphysiological modelling approach to stability in herbivore-vegetation systems. Ecol. Model., 149, 153-178.

Raia, P. \& Meiri, S. (2006). The island rule in large mammals: Paleontology meets ecology. Evolution, 60, 1731-1742.

Reznick, D.A., Bryga, H. \& Endler, J.A. (1990). Experimentally induced life-history evolution in a natural population. Nature, 346, 357-359.

Reznick, D. \& Endler, J.A. (1982). The impact of predation on life history evolution in Trinidadian guppies (Poecilia reticulata). Evolution, 160-177.

Roff, D. (1993). Evolution of life histories: Theory and analysis. Springer Science \& Business Media.

Ronget, V., Gaillard, J.-M., Coulson, T., Garratt, M., Gueyffier, F., Lega, J.-C., et al. (2018). Causes and consequences of variation in offspring body mass: Meta-analyses in birds and mammals. Biol. Rev., 93, 1-27.

Sæther, B.-E. \& Engen, S. (2015). The concept of fitness in fluctuating environments. Trends Ecol. Evol., 30, $273-281$.

Sander, P.M., Christian, A., Clauss, M., Fechner, R., Gee, C.T., Griebeler, E.-M., et al. (2011). Biology of the sauropod dinosaurs: The evolution of gigantism. Biol. Rev., 86, 117-155.

Sandvig, E.M., Coulson, T. \& Clegg, S.M. (2019). The effect of insularity on avian growth rates and implications for insular body size evolution. Proceedings of the Royal Society B, 286, 20181967.

Savage, V.M., Gillooly, J.F., Woodruff, W.H., West, G.B., Allen, A.P., Enquist, B.J., et al. (2004). The predominance of quarter-power scaling in biology. Funct. Ecol., 18, 257-282.

Schreiber, S.J. (2021). Positively and negatively autocorrelated environmental fluctuations have opposing effects on species coexistence. Am. Nat., 197, 405-414.

Smallegange, I.M., Caswell, H., Toorians, M.E. \& Roos, A.M. de. (2017). Mechanistic description of population dynamics using dynamic energy budget theory incorporated into integral projection models. Methods Ecol. Evol., $8,146-154$.

Smith, C.C. \& Fretwell, S.D. (1974). The optimal balance between size and number of offspring. Am. Nat., 108, 499-506.

Stearns, S.C. (1977). The evolution of life history traits: A critique of the theory and a review of the data. Annu. Rev. Ecol. Syst., 8, 145-171.

Stearns, S.C. (1992). The evolution of life histories. Book. Oxford University Press Oxford ; New York.

Stearns, S.C. \& Koella, J.C. (1986). The evolution of phenotypic plasticity in life-history traits: Predictions of 
reaction norms for age and size at maturity. Evolution, 40, 893-913.

Steiner, U.K., Tuljapurkar, S., Coulson, T. \& Horvitz, C. (2012). Trading stages: Life expectancies in structured populations. Exp. Gerontol., 47, 773-781.

Tavecchia, G., Coulson, T., Morgan, B.J., Pemberton, J.M., Pilkington, J., Gulland, F., et al. (2005). Predictors of reproductive cost in female soay sheep. J. Anim. Ecol., 74, 201-213.

Tilman, David. (1982). Resource competition and community structure / david tilman. Book. Princeton University Press Princeton, N.J.

Travis, J., Reznick, D., Bassar, R.D., López-Sepulcre, A., Ferriere, R. \& Coulson, T. (2014). Do eco-evo feedbacks help us understand nature? Answers from studies of the trinidadian guppy. Adv. Ecol. Res., 50, 1-40.

Tuljapurkar, S., Gaillard, J.-M. \& Coulson, T. (2009). From stochastic environments to life histories and back. Philos. T. Roy. Soc. B., 364, 1499-1509.

Tuljapurkar, Shripad. (1990). Population dynamics in variable environments. Book. Springer-Verlag New York.

Turchin, P. (1999). Population regulation: A synthetic view. Oikos, 153-159.

Watson, W. \& Walker, H. (2004). The world's smallest vertebrate, schindleria brevipinguis, a new paedomorphic species in the family schindleriidae (perciformes: gobioidei). Records-Australian Museum.

West, G.B., Brown, J.H. \& Enquist, B.J. (1997). A general model for the origin of allometric scaling laws in biology. Science, 276, 122-126.

White, T. (2008). The role of food, weather and climate in limiting the abundance of animals. Biol. Rev., 83, $227-248$.

Winkler, D.W. \& Wallin, K. (1987). Offspring size and number: A life history model linking effort per offspring and total effort. Am. Nat., 129, 708-720.

\section{Appendix}

\section{$0.1 \quad$ Linearisation of model}

We linearised the model to demonstrate that the results are not a function of the non-linear aspect of our model. They are not.

We start with the simplification provided by equation (6) which we simplify the notation of to write $1=R J E$ where $R$ is reproduction $J$ juvenile survival and $E$ is life expectancy.

We can write $R_{N}=\frac{d R}{d N}=b_{R} R$ where $b_{R}$ is the density coefficient on an exponential $R$. If $P_{a}$ is survival at age $a$ and $b_{p}$ the density coefficient on the logistic, then

$$
P_{a, N}=\frac{d P_{a}}{d N}=b_{p} P_{K}\left(1-P_{K}\right) \approx b_{p} P_{K}
$$


765

It follows that, approximately,

$$
J_{N}=\frac{d J}{d N}=b_{p} a \bar{P} J_{0}
$$

766

$767 \quad N=0$.

768 Next, we make the density effect linear,

$$
R J E=R_{0} J_{0}(1-b K) E=1
$$

769 and

$$
P_{a}=P_{a}\left(a_{\min }\right)+q\left(a-a_{m i n}\right)
$$

${ }_{772}$ If we assume survival is constant post sexual maturity at $P_{a}$ then

$$
E=\frac{1-\left[P_{a}\right]^{a}}{1-P_{a}}
$$

773 The slope of $E$ now depends upon $q$ as well as $a$, and, as in our simulations, life expectancy will only increase when ${ }_{774} q$ is large enough. We can now use values of $E, R_{0}$ and $J_{0}$ to explore how linearised $K$ varies as we change $E, b_{P}$ 775 and $b_{J}$. This is most easily done graphically. Mirroring our simulation results, divergent selection for $K$ depends on 776 a strong enough survival advantage of the delay in maturity. If not, $K$ will just fall as $a$ increases. Our results are 777 consequenlty not due to the non-linearities in our functions. 
Table 1: Parameters and variables used in models.

\begin{tabular}{|l|l|}
\hline Term & Definition \\
\hline$a$ & Age \\
\hline$a_{\mathrm{m}}$ & Age at sexual maturity \\
\hline$\beta_{0}, \alpha_{0}, \rho_{0}, \gamma_{0}$ & Intercepts of survival, development, recruitment, inheritance functions \\
\hline$\beta_{\mathrm{z}}, \alpha_{\mathrm{z}}, \rho_{\mathrm{z}}$ & Body size slopes of survival, development, recruitment functions \\
\hline$\beta_{\mathrm{N}}, \alpha_{\mathrm{N}}, \rho_{\mathrm{N}}$ & Density slopes of survival, development, recruitment function $\mathrm{s}$ \\
\hline$\alpha_{\mathrm{v}}, \gamma_{\mathrm{v}}$ & Variance terms for development, inheritance functions \\
\hline$D\left(z^{\prime} \mid z, N, t\right)$ & Inheritance function \\
\hline$E\left(a_{m}, K\right)$ & Life expectancy at sexual maturity \\
\hline $\mathrm{G}\left(z^{\prime} \mid z, N, t\right)$ & Development function \\
\hline$K$ & Carrying capacity \\
\hline $\mathbf{K}$ & Population structure at carrying capacity \\
\hline$K_{\mathrm{j}}, K_{\mathrm{a}}$ & Numbers of juveniles, adults at carrying capacity \\
\hline$L(a, K)$ & Survivorship to age $a$, evaluated at carrying capacity \\
\hline$L\left(a_{m}, K\right)$ & $\begin{array}{l}\text { Proportion of each cohort surviving to sexual maturity evaluated at } \\
\text { carrying capacity }\end{array}$ \\
\hline$\lambda$ & Population growth rate \\
\hline$N$ & Population size \\
\hline$N(z, t)$ & Distribution of body size $z$ at time $t$ \\
\hline$R 0$ & Mean lifetime reproductive success \\
\hline$R(z, N, t)$ & Recruitment function \\
\hline$R(a, K)$ & Per-capita reproductive success at age $a$, evaluated at carrying capacity \\
\hline$\theta\left(\mu=\ldots, \sigma^{2}=\ldots\right)$ & Normal distribution with mean $\mu$ and variance $\sigma^{2}$ \\
\hline$S(z, N, t)$ & Survival function \\
\hline$t$ & Time \\
\hline$z, z$, & Body size at time $t, t+1$ \\
\hline$z_{a}$ & \\
\hline
\end{tabular}


Table 2. Model parameters

\begin{tabular}{lcccc} 
Function & Intercept & $\begin{array}{c}\text { Body size } \\
\text { slope }\end{array}$ & $\begin{array}{c}\text { Density } \\
\text { slope }\end{array}$ & $\begin{array}{c}\text { Variance } \\
\text { intercept }\end{array}$ \\
\hline Survival parameters scenario 1 & -0.875 & 0.15 & 0 & \\
Survival parameters scenario 2 & 0.25 & 0.125 & -0.001 & \\
Reproduction parameters scenario 1 & 1 & 0 & -0.001 & \\
Reproduction parameters scenario 2 & -1 & 0 & 0 & 1 \\
Growth parameters & 6.8 & 0.3 & 0 & 1 \\
& 6.69 & 0.33 & 0 & 1 \\
& 6.59 & 0.35 & 0 & 1 \\
Offspring parameters & & & & 1
\end{tabular}



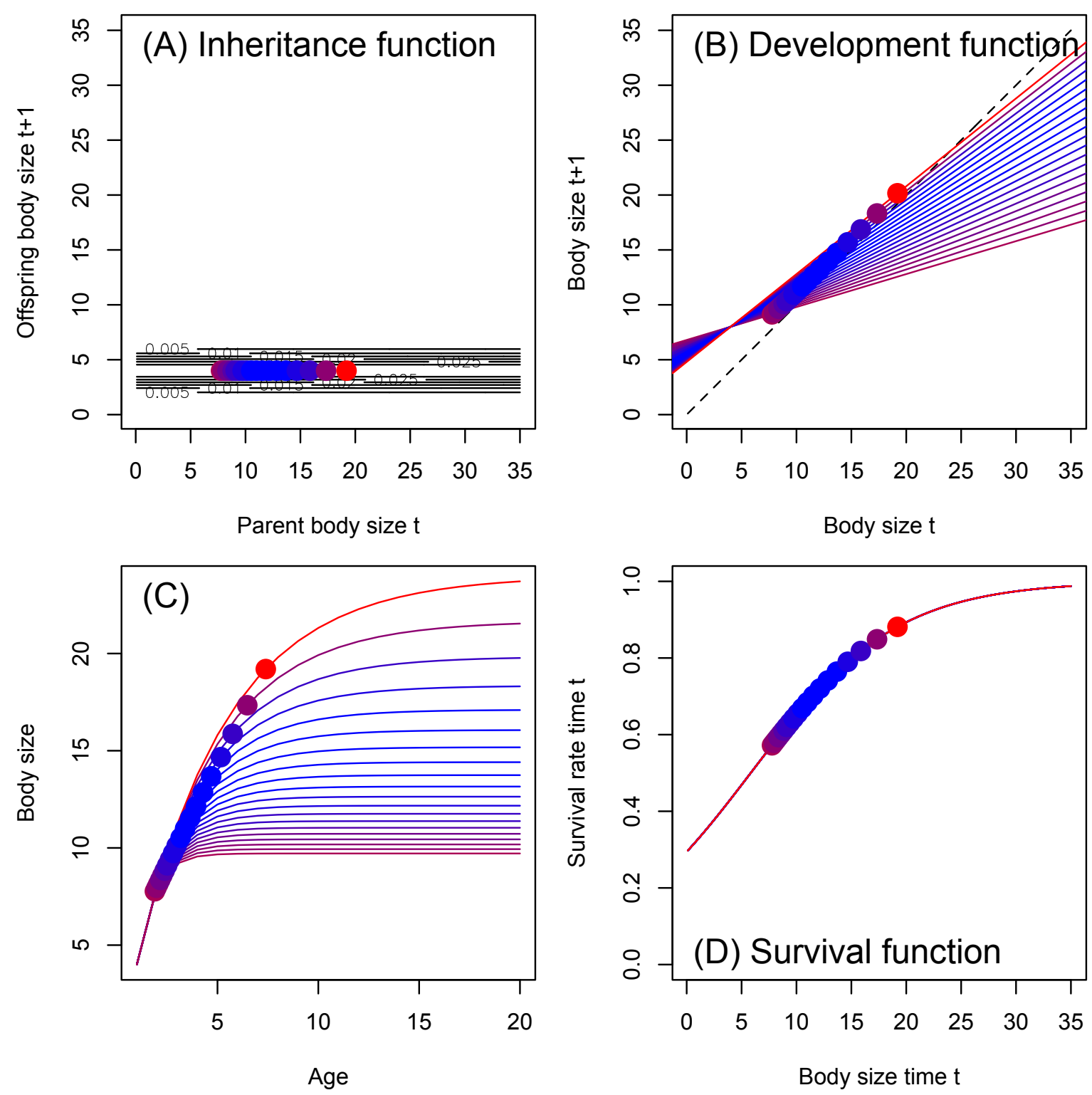

Figure 1. Density-independent functions in scenario 1. (A) association between parental size at time $t$ and offspring size at time $t+1,(B)$ development functions, (C) monomolecular growth functions, (D) body sizesurvival function. Each point represents one of our 20 life histories. The redder the colour of a point, the fitter the life history strategy. 


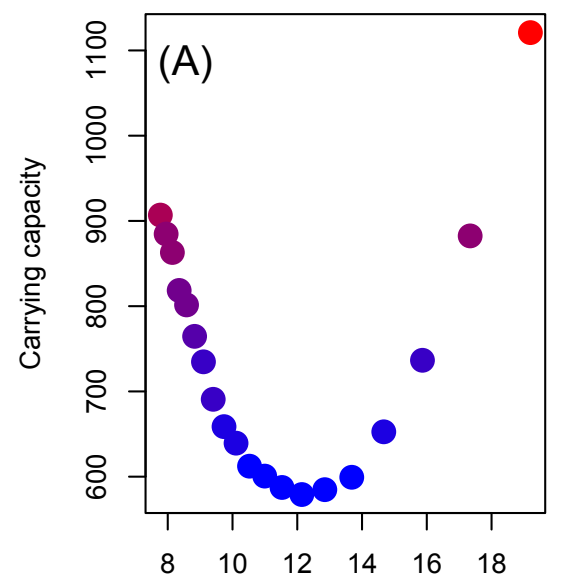

Size at sexual maturity

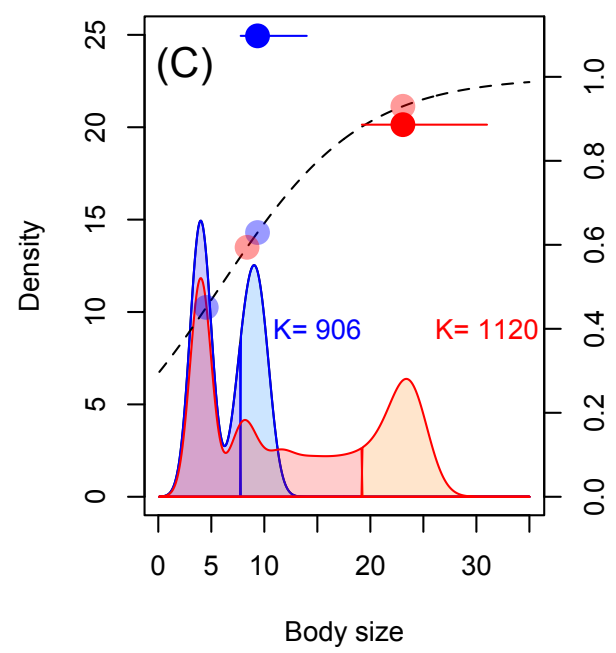

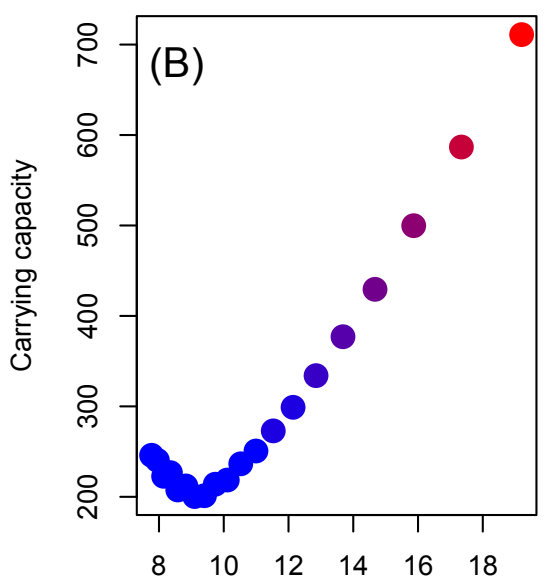

Size at sexual maturity

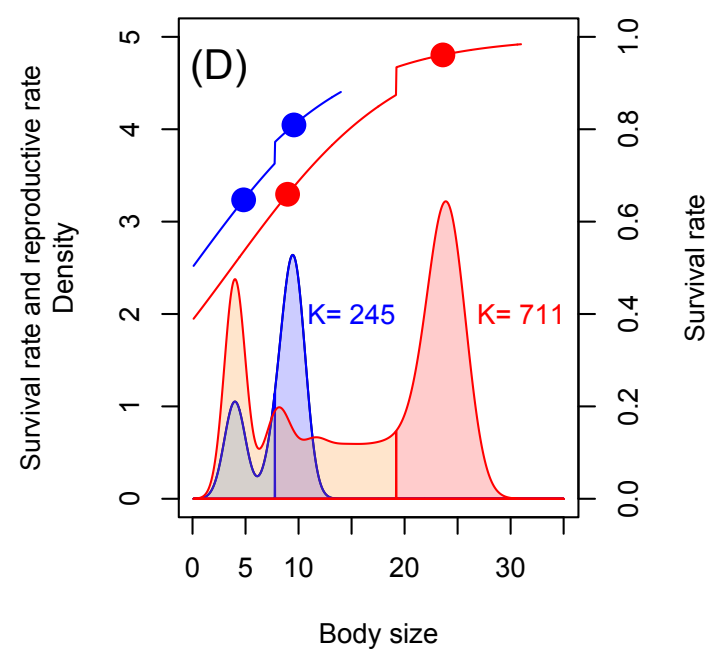

Figure 2. Scenario outcomes and why different life histories have different carrying capacities at equilibrium. Disruptive selection on size at sexual maturity for (A) scenario 1 and (B) scenario 2. Each point represents one of our 20 life histories. The redder the colour of a point, the fitter the life history strategy. (C) In scenario 1, the stable size distribution differs between life histories (polygons). Two life histories are shown - one shaded blue (small size, fast life history, lower fitness) and the other in red (large size, slow life history, higher fitness). Vertical lines separate juveniles from adults. The different development rates across life histories result in different juvenile and adult survival rates (pastel dots on dashed black line) despite both strategies being defined with identical body size-survival functions. The higher survival rates in the slower life history allow a lower rate of per-capita reproduction (horizontal lines and brightly coloured dots) and this is achieved via a higher carrying capacity (red and blue numbers). The models produce the observed stable size-structures. (D) a version of (C) for scenario 2. Juvenile survival is density-dependent while the reproductive rate is constant across life histories (not shown). The slower life history has a higher carrying capacity $(K=711)$ than the faster one $(K=245)$ and consequently has a lower elevation of the survival function (compare the heights of the blue and red curves at low values of body size). However, because juvenile and adult size is larger in the slower life history, juvenile and adult survival rates are also higher than for the slower life history (compare red and blue dots which show survival rates for mean juvenile and mean adult body size). In (C) and (D) the solid points represent density-dependent rates. 

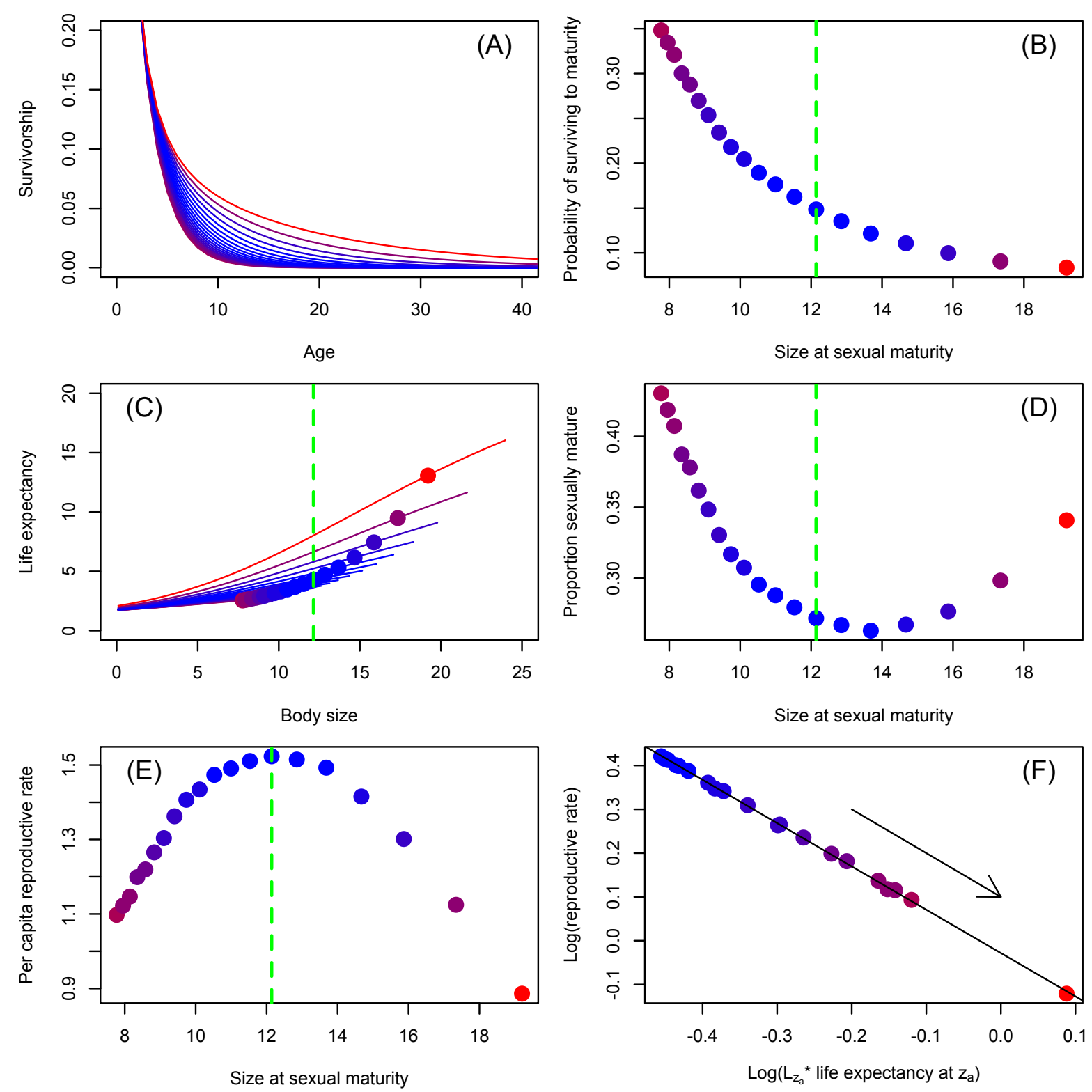

Figure 3. (A) survivorship functions for each life history, (B) survivorship to sexual maturity as a function of size at sexual maturity, (C) life expectancy as a function of body size, (D) proportion of population that is sexually mature as a function of size at sexual maturity, and (E) per-time step per-capita reproductive rate as a function of size at sexual maturity, and (F) trade-off between the log of the densityindependent rates with the log of the per-capita per-time step reproductive rate (the arrow represents the direction of evolution). The dotted green vertical lines in (B-E) represent the life history of minimum fitness. Each point represents one of our 20 life histories. The redder the colour of a point, the fitter the life history strategy. 
(A) Age 4 at sexual maturity

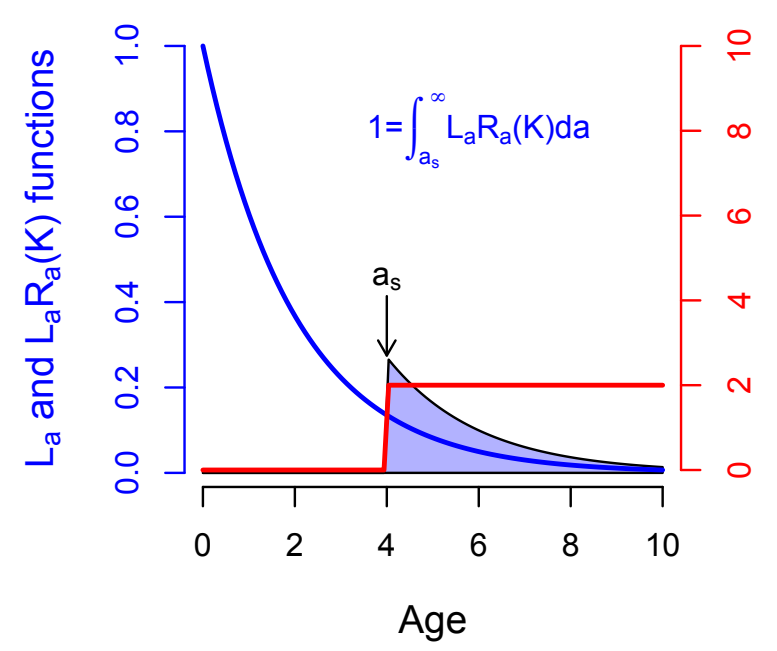

(C) Rectangular depiction

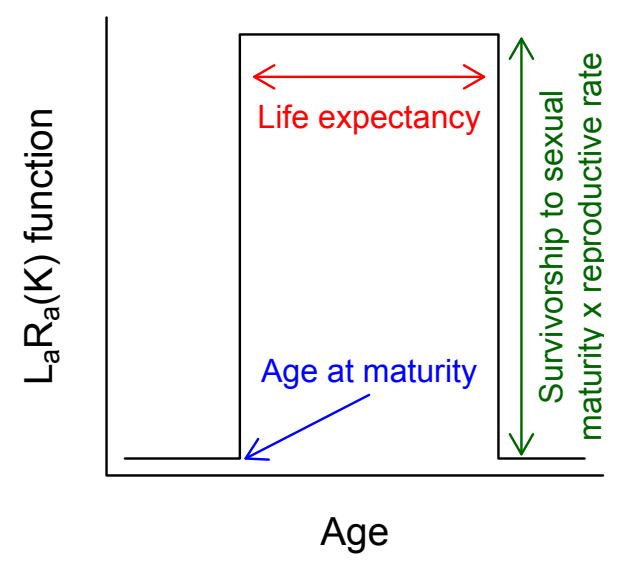

(B) Age 5 at sexual maturity

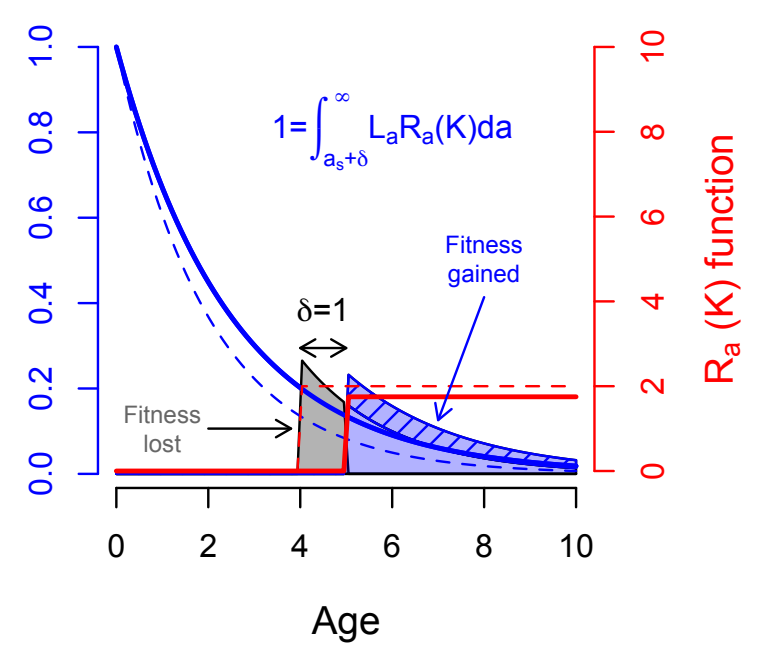

Figure 4. Summary of the age-structured life history dynamics of the model where the reproduction function is density-dependent as in Scenario 1. The initial life history strategy is depicted in $(A)$, the mutant strategy, with a delayed age at sexual maturity, in (B). The delay in age at sexual maturity results in a change in the development function (see Figure $1(B, C)$ ) that results in an elevation of the survivorship function (compare the solid blue line in (B) to the solid blue line in $(A)$ which is also represented by the dotted blue line in (B)). Because the volume of the blue polygon in $(A)$ and $(B)$ must equal unity (equations on plot), the reproduction function is depressed in (B) compared to ( $A$ ) (compare the solid red lines in (A) and (B)). The grey and hashed blue polygons in (B) show how the polygon has changed shape between the two life histories. $(C)$ Rectangular approximation of the life history function used to identify trade-offs. 

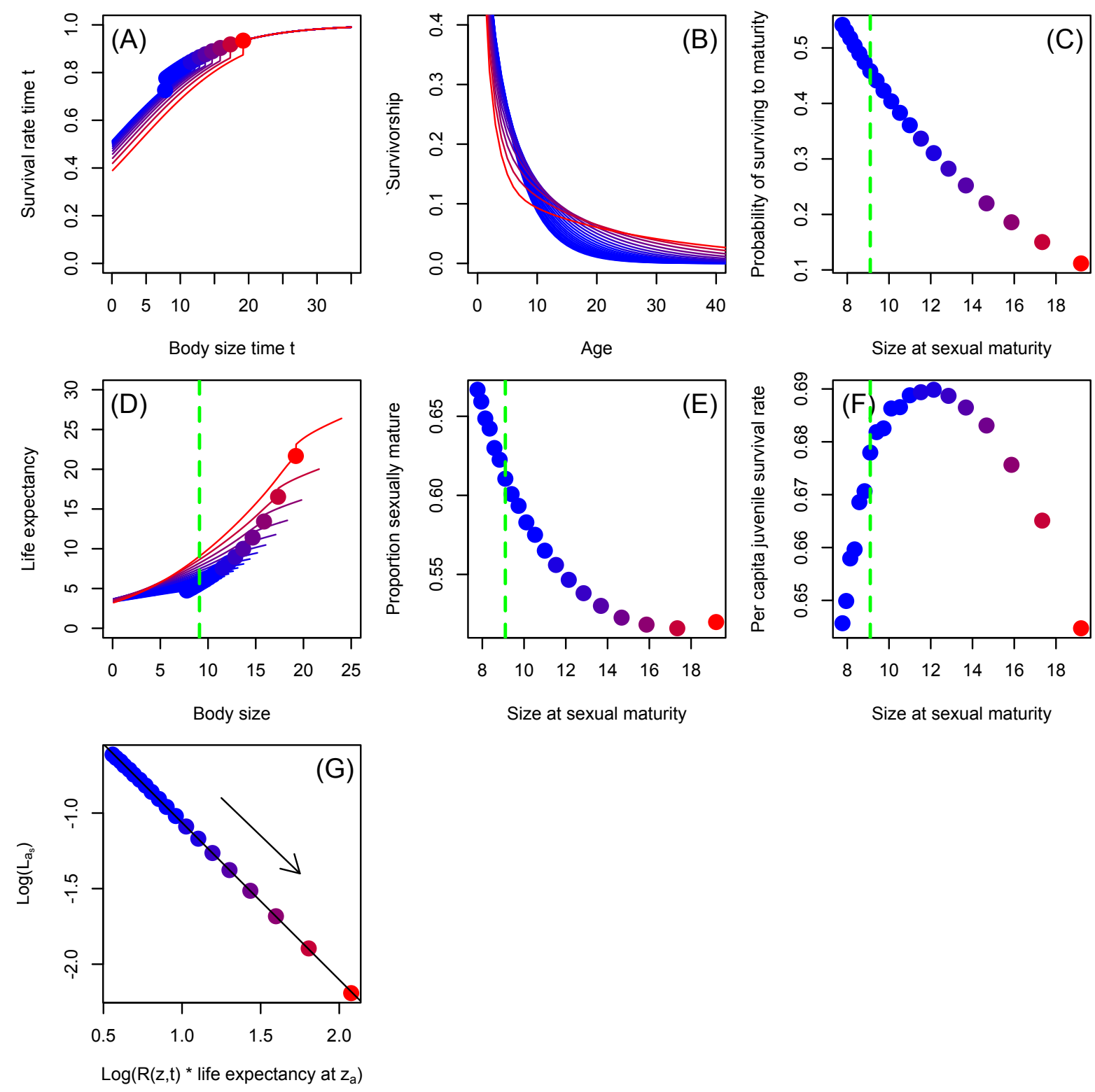

Figure 5. Model structure and outputs when the juvenile survival function is density-dependent (scenario 2). (A) body size-survival function, (B) survivorship functions for each life history, (C) survivorship to sexual maturity as a function of size at sexual maturity, $(D)$ life expectancy as a function of body size, (E) proportion of population that is sexually mature as a function of size at sexual maturity, and (F) per-time step per-capita juvenile survival rate as a function of size at sexual maturity for each life history. (G) linear associations between the log of the density-independent rates against the log of the densitydependent rate. Each point represents one of our 20 life histories. The redder the colour of a point, the fitter the life history strategy. 


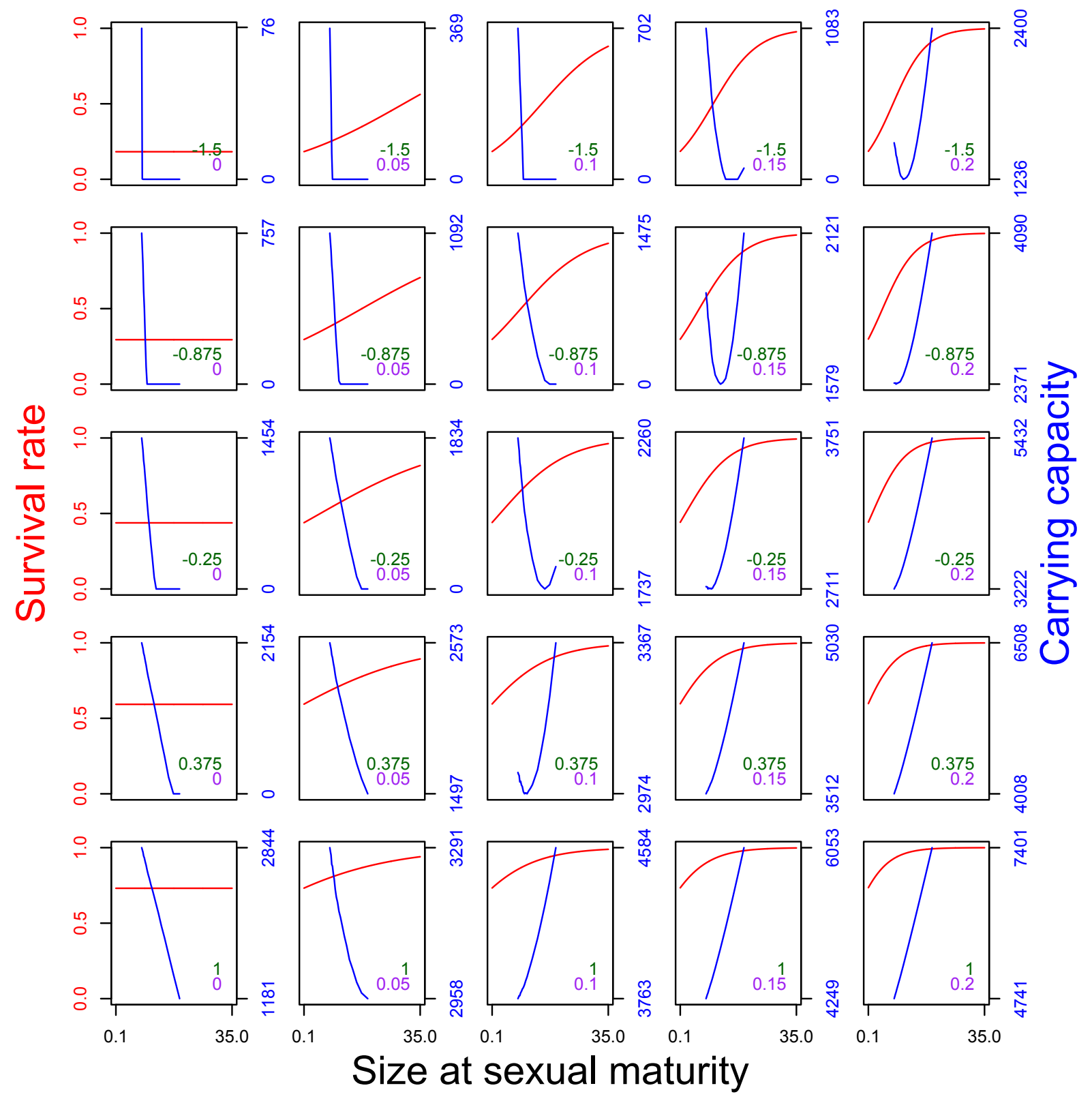

Figure 6. Dynamical consequences of altering the intercept and slope of the body size-survival function. As the elevation of the intercept (rows, and green numbers) and steepness of slope (columns and purple numbers) are altered, the change in the size-survival function (red lines) alters selection on size at sexual maturity (blue lines). 


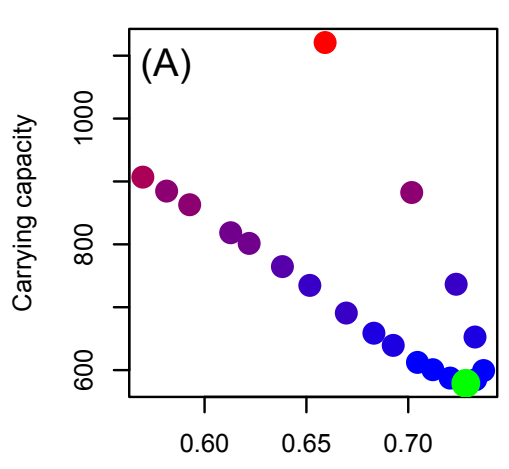

Proportion juvenile

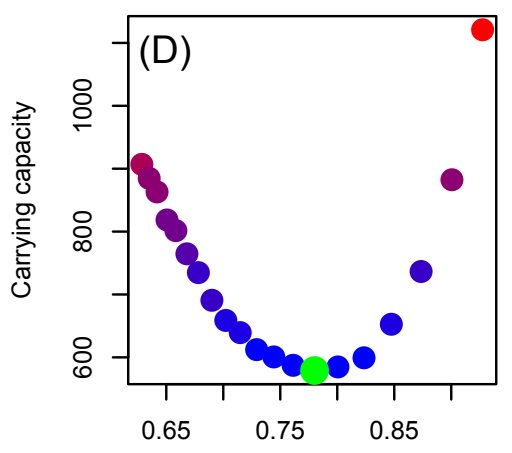

Mean adult survival

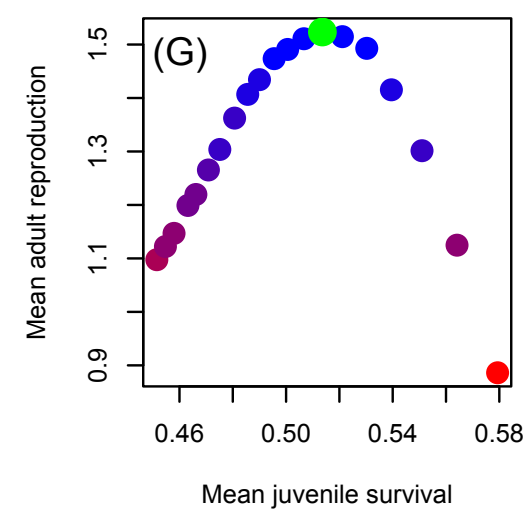

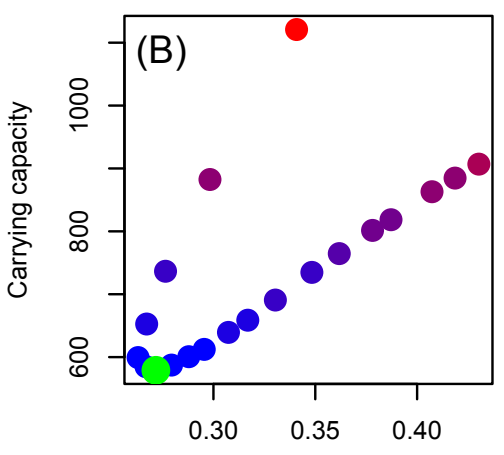

Proportion adult
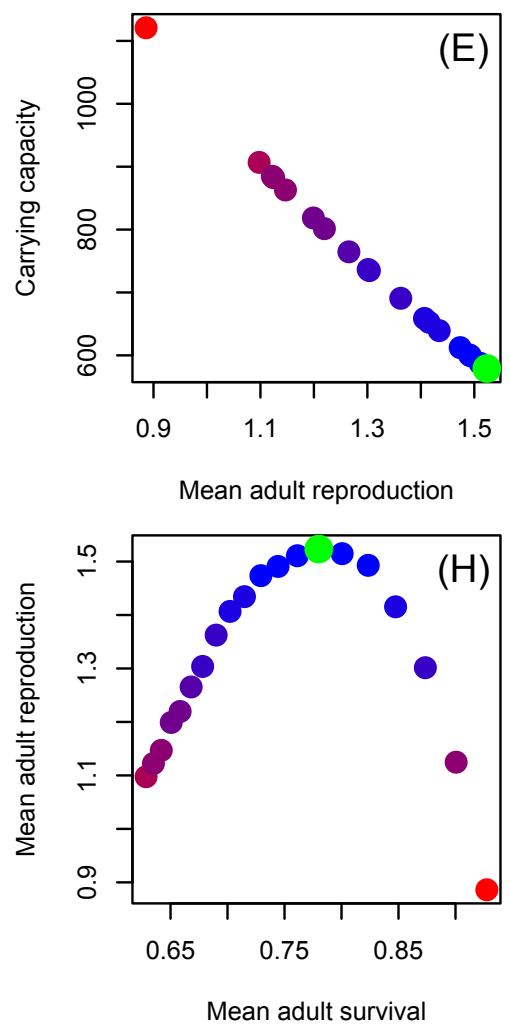
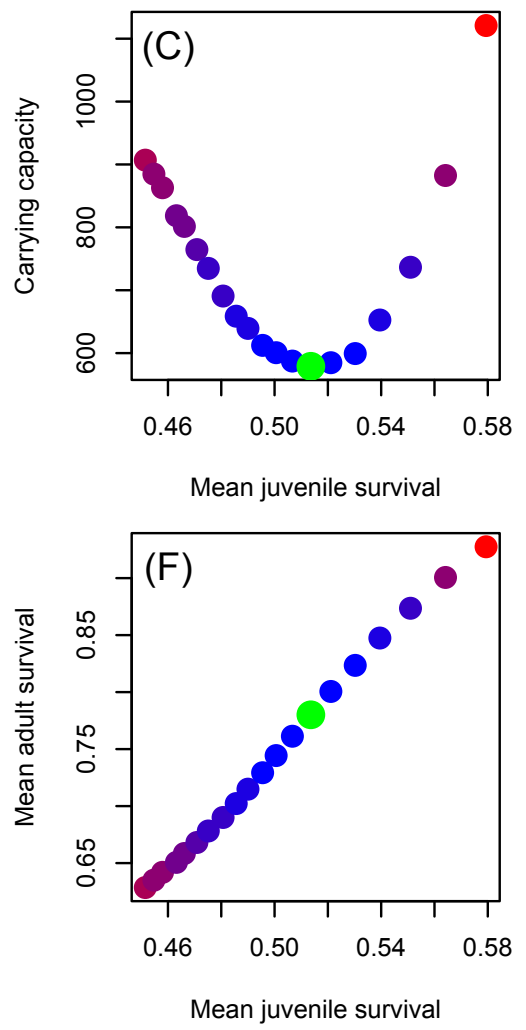

Figure S1. Associations across life histories and between the terms that determine carrying capacity and carrying capacity for the model with density dependence in reproduction. (A) population size of juveniles, (B) population size of adults, (C) mean juvenile survival rate, (D) mean adult survival rate, $(E)$ mean reproductive rate, $(F)$ population size of juveniles $x$ mean juvenile survival rate, $(G)$ population size of adults $x$ mean adult survival rate, $(H)$ population size of adults $x$ recruitment rate, $(H)$ population size of adults $x$ (mean adult survival + mean reproductive rate). Blue dots represent fast life histories, red dots slower ones. Each point represents one of our 20 life histories. The redder the colour of a point, the fitter the life history strategy. 

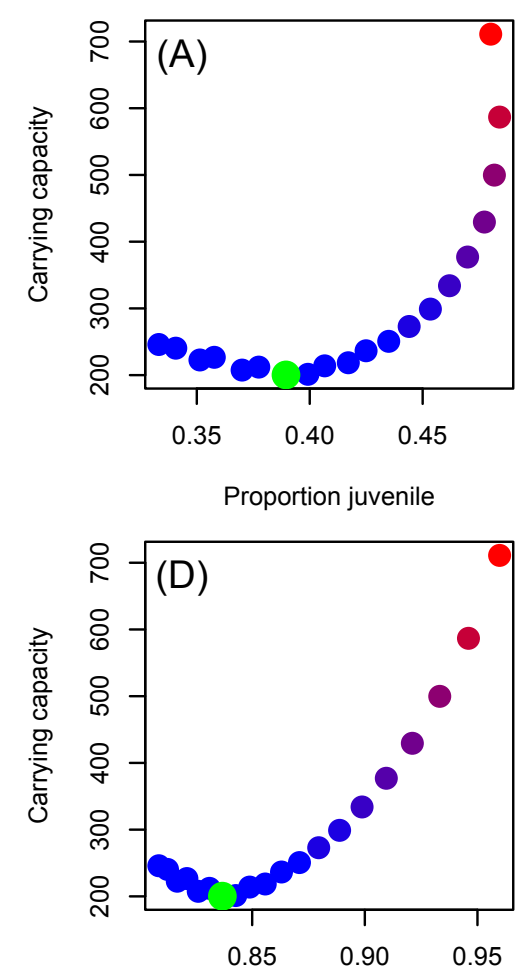

Mean adult survival

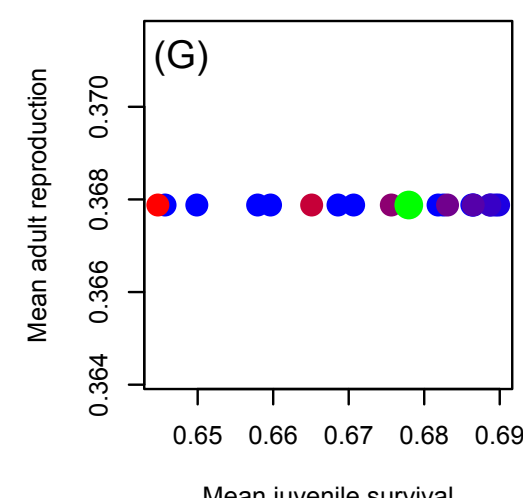

Mean juvenile survival
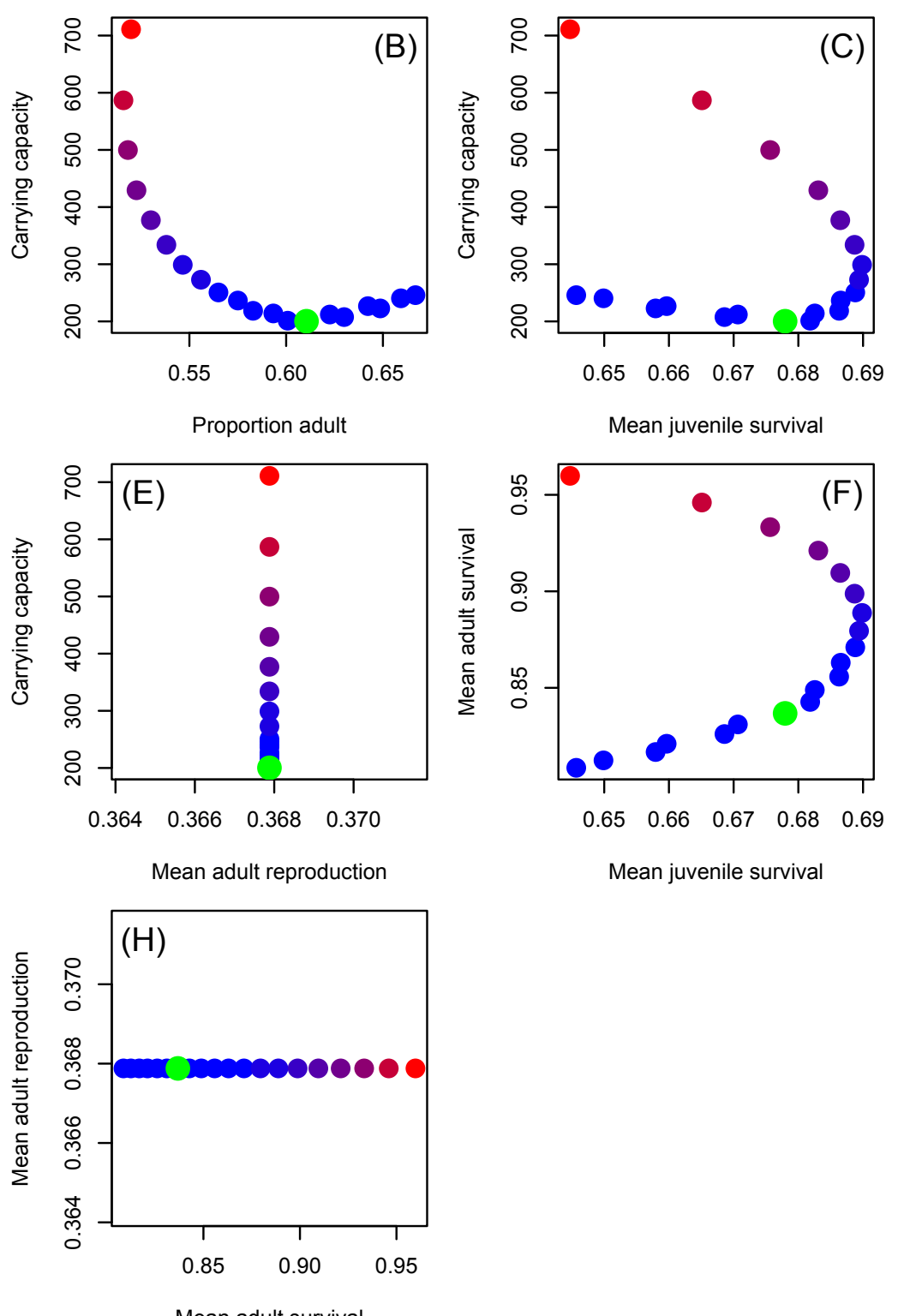

Figure S2. Association across life history between the terms that determine carrying capacity and carrying capacity for the model with density dependence in juvenile survival. (A) population size of juveniles, $(B)$ population size of adults, $(C)$ mean juveniles survival rate, $(D)$ mean adult survival rate, $(E)$ mean reproductive rate, $(F)$ population size of juveniles $x$ mean juvenile survival rate, (G) population size of adults $x$ mean adult survival rate, $(H)$ population size of adults $x$ recruitment rate, $(H)$ population size of adults $x$ (mean adult survival + mean reproductive rate). Blue dots represent fast life histories, red dots slower ones. Each point represents one of our 20 life histories. The redder the colour of a point, the fitter the life history strategy. 
bioRxiv preprint doi: https://doi. org/10.1101/2022 02 17.480952. this version posted February 18,2022 . The copyright holder for this preprint (which was not certified by peer review) is the author/funder, who has granted bioRxiv a license to display the preprint in perpetuity. It is made available under aCC-BY-NC-ND 4.0 International license.
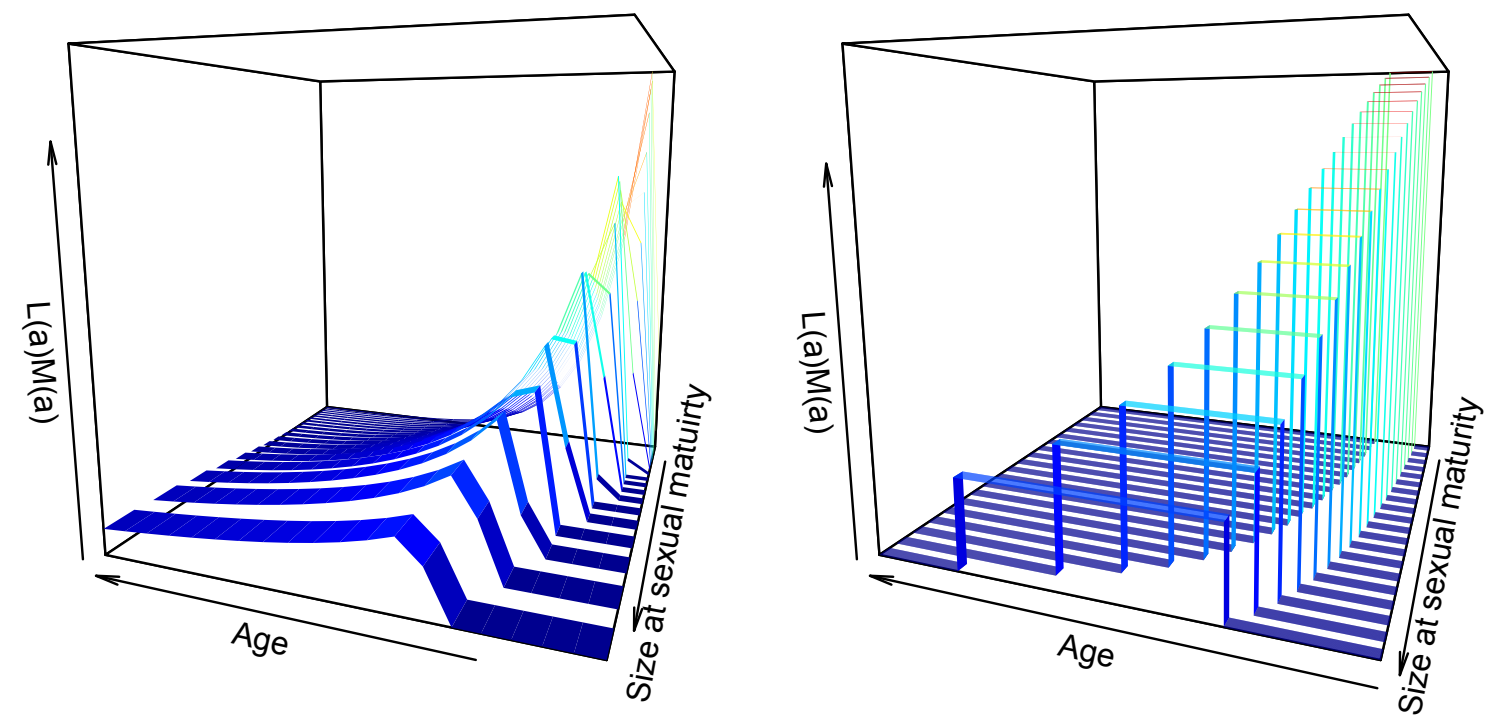

Figure S3. The life history functions for each of the 20 life histories for scenario 1 (left), along with the rectangular approximation we use (right). See figure 4(C) for an additional description of this approximation. 